\title{
Heliospheric evolution of solar wind small-scale magnetic flux ropes
}

\author{
M. L. Cartwright ${ }^{1}$ and M. B. Moldwin ${ }^{2}$ \\ Received 18 March 2009; revised 26 March 2010; accepted 6 April 2010; published 10 August 2010.
}

[1] We present results from the first comprehensive small-scale flux rope survey between 0.3 and 5.5 AU using the Helios 1, Helios 2, IMP 8, Wind, ACE, and Ulysses spacecrafts to examine their occurrence rate, properties, and evolution. Small-scale flux ropes are similar to magnetic clouds and can be modeled as a constant-alpha, force-free, cylindrically symmetric flux rope. They differ from magnetic clouds in that they have durations on the order of tens of minutes up to a few hours, they lack an expansion signature at $1 \mathrm{AU}$, and they do not have a depressed proton temperature compared to the surrounding solar wind plasma. The occurrence rate of small-scale flux ropes is slightly higher in the inner heliosphere than the outer heliosphere and has a weak dependence on the phase of the solar cycle. The duration of the events as a function of radial distance indicates there is a large, rapid expansion within $1 \mathrm{AU}$ and it becomes constant in the outer heliosphere. This behavior implies small-scale flux ropes are created and nearly complete their evolution within $1 \mathrm{AU}$.

Citation: Cartwright, M. L., and M. B. Moldwin (2010), Heliospheric evolution of solar wind small-scale magnetic flux ropes, J. Geophys. Res., 115, A08102, doi:10.1029/2009JA014271.

\section{Introduction}

[2] Magnetic flux ropes are a common phenomenon in the heliosphere. They are created on the Sun, in the Earth's magnetotail, and at other planets. They can be described as a bundle of magnetic field lines twisted into a tube-like shape with a strong axial field. In the outer section of the rope, the twisted field is strongest while the axial field lines become dominant in the inner section. In cylindrical coordinates, the axial field is called the core field of the flux rope and the azimuthal field is called the twist or bipolar field of the flux rope.

[3] Magnetic flux ropes are important because they transport field and plasma from one location to another. The scale size of a flux rope is dependent on the environment in which it was created and its subsequent expansion. For example, in the Earth's magnetotail, flux ropes have scale sizes on the order of $17 R_{E}$. They are commonly believed to be created by magnetic reconnection across the magnetotail plasma sheet [Moldwin and Hughes, 1992].

[4] A large-scale flux rope originating at the Sun is called a magnetic cloud (MC) and has been defined as: (1) a smooth magnetic field rotation parallel to a plane, (2) enhanced magnetic field strength compared to the surrounding solar wind, and (3) a depressed proton temperature [Burlaga et al., 1981]. These events have been well-studied from 0.3 to

\footnotetext{
${ }^{1}$ Space Sciences Laboratory, University of California, Berkeley, California, USA.

${ }^{2}$ Department of Atmospheric, Oceanic, and Space Sciences, University of Michigan, Ann Arbor, Michigan, USA.

Copyright 2010 by the American Geophysical Union. 0148-0227/10/2009JA014271
}

5.5 AU [e.g., Klein and Burlaga, 1982; Bothmer and Rust, 1997; Bothmer and Schwenn, 1998; Mulligan et al., 1998; Lepping et al., 1990, 2006; Lynch et al., 2005; Lepping and $\mathrm{Wu}, 2007]$. They are on average $21 \mathrm{~h}$ in duration, varying between 8 and $48 \mathrm{~h}$ at $1 \mathrm{AU}$. The axial field strength is typically greater or equal to twice the background field. The occurrence frequency per year has a low correlation with the monthly sunspot number, based on observations from 1995 to 2003. At $1 \mathrm{AU}, \mathrm{MCs}$ usually have a decreasing velocity profile across the duration of the event. This implies that they are still expanding into the solar wind and are not in pressure balance [e.g., Lepping et al., 2006].

[5] The most utilized flux rope model is called the static constant-alpha, force-free, cylindrically symmetric field geometry [i.e., Goldstein, 1983; Burlaga, 1988]. The forcefree condition implies there are no gravitational or thermal pressure gradient forces and the structure is in steady state allowing $\nabla \times \vec{B}=\mu_{0} \vec{J}=\alpha \vec{B}$. Assuming alpha is constant, Lundquist [1950] showed this equation has solutions of Bessel functions as seen in equation 1.

$$
\begin{array}{cc}
B_{R}=0 & \text { Radial component } \\
B_{T}=B_{0} H J_{1}(\alpha R) & \text { Tangential component } \\
B_{A}=B_{0} J_{0}(\alpha R) & \text { Axial component }
\end{array}
$$

where $\mathrm{B}_{0}$ is the field magnitude on the axis, $R=r / R_{0}$ where $R_{0}$ is the radius of the cloud, and $r$ is the radial distance from the cloud axis. $J_{0}$ and $J_{1}$ are the zeroth- and first-order Bessel functions. $H= \pm 1$ and defines the sign of magnetic 

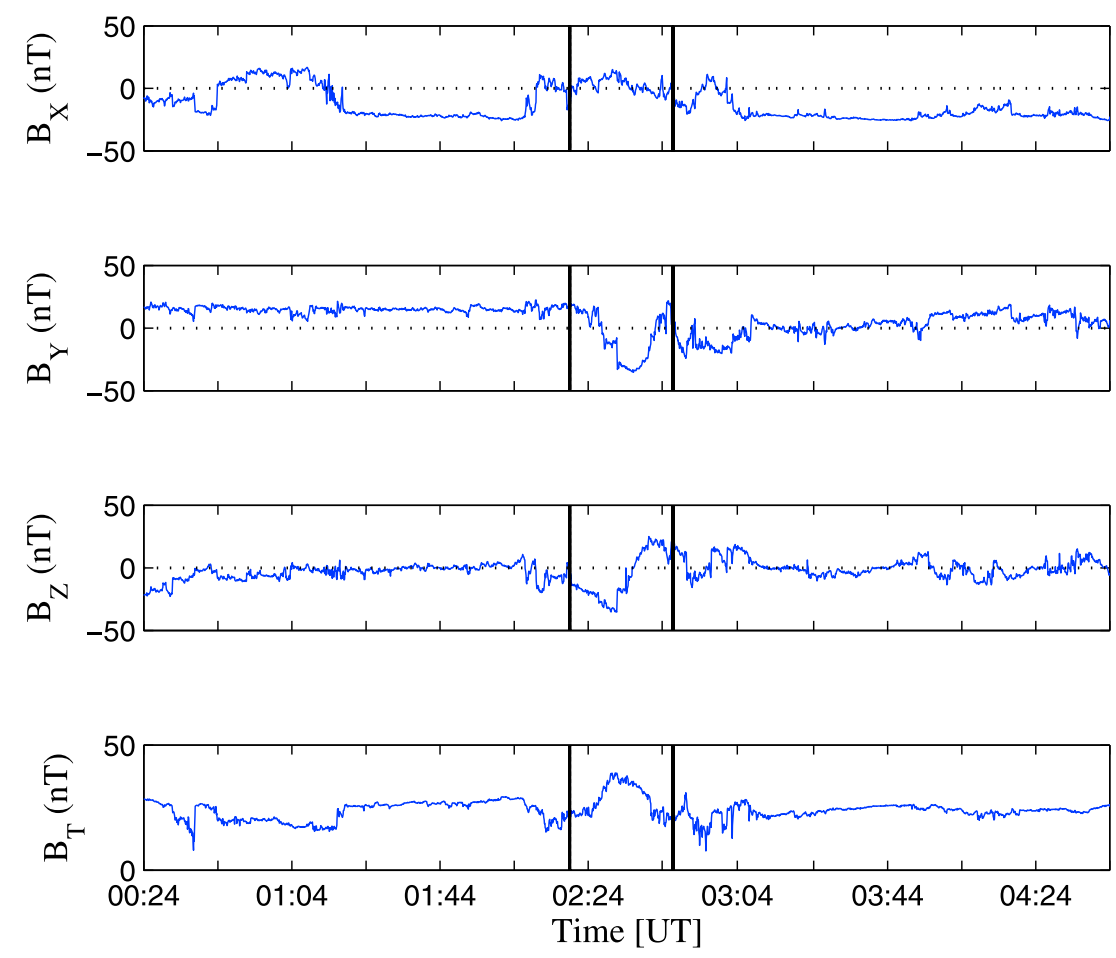

Figure 1. An example of a small-scale flux rope found at 0.39 AU. It was identified from the Helios 1 data set on 4 March 1975 from 0219 to 0247 UT. The black vertical bars indicate the boundaries of the event.

helicity of the flux rope. The flux rope boundaries are defined to be the first zero of the zeroth Bessel function, where $r=R_{0}$ and the value of $\alpha$ is 2.40 . The magnetic helicity is a measure of how twisted the field has become and the handedness. Many interplanetary coronal mass ejections (ICMEs) will not fit the classic $\mathrm{MC}$ criteria and require more advanced models. If the structure is not force-free or cylindrically symmetric, then the use of a symmetric, static model will give large errors and many interesting events will be excluded from the data set, e.g., the MC-like events from the work of Lepping et al. [2006]. There have been models focused on cases where alpha is not constant [e.g., Farrugia et al., 1995; Osherovich et al., 1999; Vandas et al., 1993; Mulligan, 2002] but such models are often designed to model the magnetic cloud evolution. They are not valid at particular stages of evolution.

[6] MCs are large structures and it is common to study them using hourly data sets. However, hourly averages cannot be used to study phenomena with timescales of the order of tens of minutes to hours. The solar wind small-scale flux ropes identified by Moldwin et al. [1995, 2000] are such a phenomenon. These structures were first discovered by Moldwin et al. [1995] with the Ulysses spacecraft near 5 AU. The one event identified was characterized by a bipolar field rotation coincident with a strong axial core field with duration of $4 \mathrm{~h}$. This event motivated a small survey at 1 AU for small-scale flux ropes, where Moldwin et al. [2000] identified six events with the Wind and IMP 8 magnetic field data sets. These events all have bipolar field rotations coincident with a core field enhancement and were on the order of tens of minutes duration and displayed the signature of a force-free, symmetric magnetic flux rope.
[7] Small-scale solar wind flux ropes have recently been extensively studied at 1 AU [Cartwright and Moldwin, 2008; Feng et al., 2007, 2008]. A classic example of a small-scale flux rope (as shown in Figure 1) was observed by Helios 1 at $0.39 \mathrm{AU}$. The magnetic field vectors are shown in solar ecliptic (SE) coordinates. This is a heliocentric coordinate system where $B_{x}$ points from the spacecraft to the Sun, $B_{z}$ is normal to and northward from the ecliptic plane, and $B_{y}$ completes the right-handed set. The magnetic field topology is similar to a magnetic cloud, where in Figure 1 the core field is in $B_{Y} \mathrm{SE}$ coincident with a bipolar field observed in $B_{z}$ SE component. The $B_{x} \mathrm{SE}$ component is flat consistent with the Lundquist solution shown in equation (1).

[8] Given that the magnetic field topology is similar to magnetic clouds, the search parameters used to identify small-scale flux ropes involved strong core fields associated with a bipolar field in one of the components. The studies by Feng et al. [2007, 2008] visually identified events from the years 1995 to 2005 and the study by Cartwright and Moldwin [2008] used an automated technique to identify events from 1995 to 2005 . There was little overlap between the lists because the automated technique [Cartwright and Moldwin, 2008] quantitatively searched for strong core fields. The majority of the events identified by the visual survey by Feng et al. [2007] had weaker core field peaks than the automated survey identified. Upon further examination by Cartwright and Moldwin [2008], these weaker core field flux ropes found by Feng et al. [2007] were often consistent with compressive Alfvén waves. At these smallscale sizes, there are solar wind disturbances called com- 
pressive Alfvén waves (or fast mode waves) that can have enhanced total field intensity and a similar magnetic field signature [Burlaga and Turner, 1976]. These events are identified by a cross correlation of the magnetic field and velocity field vectors due to the Alfvén wave property that for an isotropic plasma, $\mathbf{v}= \pm\left(V_{A} / B_{0}\right) \mathbf{b}$. The vectors $\mathbf{v}$ and $\mathbf{b}$ are the perturbations of velocity and magnetic field, $B_{0}$ is the average field strength, and $V_{A}$ is the Alfvén speed defined to be: $V_{A}=B /\left(\mu_{0} \rho\right)^{1 / 2}$. Studies investigating small-scale flux ropes should exclude these events because the magnetic signatures are identical.

[9] The comprehensive surveys at $1 \mathrm{AU}$ [Cartwright and Moldwin, 2008; Feng et al., 2007; 2008] indicated a population of small-scale flux ropes that dominates in occurrence frequency over large-scale flux ropes (or MCs) identified from the Lepping et al. [2006] database and maintained online at http://lepmfi.gsfc.nasa.gov/mfi/mag_cloud pub1. html. These three databases [Cartwright and Moldwin, 2008; Feng et al., 2007, 2008; Lepping et al., 2006] all used Wind data that included some data gaps. Although the magnetic topology of small-scale flux ropes is similar to MCs, these structures differ from MCs in several key ways. Small-scale flux ropes have durations on the order of tens of minutes to several hours and scale sizes on the order of a few hundred Earth radii (0.001-0.003 AU), where MCs are on the order of $20 \mathrm{~h}$ and a quarter of an AU in size. Small-scale flux ropes have constant temperature profiles similar to the surrounding solar wind, unlike MCs that have depressed proton temperatures. At $1 \mathrm{AU}$, they show little expansion unlike MCs, indicating they are at their evolutionary endpoint [Cartwright and Moldwin, 2008]. These differences suggest that they may have different origins, with MCs formed in the lower corona and small-scale magnetic flux ropes forming in the solar wind.

[10] These past surveys at $1 \mathrm{AU}$ and the differences from MCs motivate the current study of the radial evolution of small-scale magnetic flux ropes in the solar wind from 0.3 to 5.5 AU. Ideally, to study the evolution of a small-scale flux rope, the same event should be examined with a spacecraft at various heliospheric distances. We searched the various data sets for such an event but found no event observed by widely separated spacecrafts between 0.3 and 1 AU or 1 and 5.5 AU. This survey presents the results of small-scale flux ropes identified at various heliospheric distances to understand the collective behavior of these objects from the inner to middle heliosphere. This study answers the questions: Are these small-scale flux ropes observed at all radial distances from the Sun? Do they have the same properties at all distances? Do the answers to the questions provide clues to their origins and formation mechanism?

\section{Methodology}

\subsection{Spacecraft Used in This Survey}

[11] We examined Helios 1, Helios 2, IMP 8, Wind, ACE, and Ulysses magnetic field and plasma data sets available from the National Space Science Data Center, the Space Physics Data Facility, and the solar wind data set compiled to develop a solar wind data set (propagated to the bow shock or not propagated) by J. Weygand at UCLA (http:// www.igpp.ucla.edu/jweygand/htmls/Propagated_SW.html).
[12] Helios 1 and Helios 2 were launched in December 1974 and January 1976, respectively, into a heliocentric orbit that ranged from 0.3 to $1 \mathrm{AU}$. For more information on the instrumentation and data analysis of the magnetometer experiment, see the work of Scearce et al. [1975], and for the plasma experiment, see the work of Rosenbauer et al. [1977]. The magnetic field data are available from 15 December 1974 to 16 June 1981 in SE spacecraft-centered coordinates and contain many data gaps.

[13] IMP 8 was one of a series of probes used to investigate Earth's magnetosphere and the solar wind. The IMP 8 data used in this study were from 1975 to 1994 , where the spacecraft was in an approximate 12.5 day geocentric orbit. This time period was used to extend the data set between Helios and the start of the Wind mission and provide a $1 \mathrm{AU}$ set of observations that overlap with the Helios data over a solar cycle. IMP 8 was in the solar wind from 7 to 9 days out of the 12.5 orbit. The data coverage in the solar wind was around $60 \%$ but contained many small (approximately tens of minutes) data gaps, which decreased the usable coverage for locating small flux ropes to around $45 \%$, depending on the year. The Goddard Space Flight Center magnetometer and the Massachusetts Institute of Technology and Los Alamos National Laboratory plasma data sets used in this study were in geocentric SE (GSE) coordinates at a time resolution of $1 \mathrm{~min}$.

[14] The Wind spacecraft was launched in November 1994 to measure the solar wind and Earth's foreshock. Following a double lunar swingby, Wind was placed in a halo orbit around the first Lagrangian (L1) point allowing for sampling of the incoming solar wind at Earth and spent time in petal orbits near the Earth and the Moon. The magnetic field and plasma data sets used were when Wind was in the solar wind and are described by Lepping et al. [1995] and Ogilvie et al. [1995], respectively. We used data in GSE coordinates at a 1 min resolution for years 1995 to 2007.

[15] ACE was launched in August 1997 as a solar wind monitor at L1. We used data from 1997 to 2007 in GSE coordinates at 1 min time resolution. For more information on the magnetic and plasma experiments, see the work of Smith et al. [1998] and McComas et al. [1998].

[16] Ulysses was launched in October 1990 with the primary purpose to investigate the solar wind, neutral gases, and energetic particles as a function of solar latitude. After launch, Ulysses cruised to Jupiter in the ecliptic plane and used the Jupiter flyby to achieve a heliospheric orbit over the solar poles. We will only present results of small-scale flux ropes found in the ecliptic plane. At higher latitudes, out of the ecliptic plane, the solar wind is composed almost entirely of the fast solar wind $\left(>600 \mathrm{~km} \mathrm{~s}^{-1}\right)$. Within the fast solar wind shear, Alfvén waves are found to be almost continuous [Smith et al., 1995] from less than $1 \mathrm{~h}$ up to $10 \mathrm{~h}$ in scale size [Bruno et al., 1985]. These waves are at the same time and length scales as small-scale flux ropes and share the same magnetic field signature of a bipolar field. This problem will limit the number of small-scale flux ropes found in the fast wind. Because of the Alfvénic nature of the fast wind, there will be a bias in the selection of small-scale flux ropes toward being in the slow wind. Because of the similarities in the magnetic signature and the near-continuous spectrum of these Alfvén waves, identifying small-scale 
flux ropes is extremely difficult out of the ecliptic plane. We used data from when Ulysses was in cruise to Jupiter and the three subsequent aphelion passes at $5.4 \mathrm{AU}$ (within $\pm 10^{\circ}$ of the solar ecliptic plane, which is $2.9^{\circ}$ and $-17.2^{\circ}$ in heliographic latitude). These passes were during the time periods 5 March 1992 to 17 September 1992, 18 October 1997 to 27 November 1998, and 26 December 2003 to 30 January 2005. Information on the magnetic and plasma data experiments can be found in the work of Balogh et al. [1992] and Bame et al. [1992]. In this study, the data were in radialtangential-normal (RTN) coordinates at 1 (4) min time resolution for the magnetic (plasma) data, respectively.

\subsection{Selection Criteria}

[17] The small-scale flux rope selection was done in three parts: (1) a visual survey of the solar wind magnetic field data sets, (2) a removal of compressive Alfvén waves, and (3) a model validation of the events.

[18] We visually examined the magnetic field data sets using an approximate $10 \mathrm{~h}$ window. We decided to proceed with a visual survey over an automated identification method [Cartwright and Moldwin, 2008] primarily because it was difficult to adapt the program to manage the data gaps from the Helios and IMP 8 data sets. We wanted to survey all the data sets in the same manner, so we used a visual survey with a wave removal method for this study. We used the force-free cylindrically symmetric flux rope property of an inflection point located at or near the peak of the axial core field in the small-scale flux rope [Moldwin et al., 2000]. We first identified an inflection point in the $B_{Y}$ or $B_{Z}$ coordinate coincident with a core field peak in the remaining component $\left(B_{Y}\right.$ or $\left.B_{Z}\right)$. The boundaries of an ideal magnetic flux rope are current sheets that often can be observed as minima in the total field component and/or a directional discontinuity separating the flux rope structure from the surrounding solar wind plasma. We identified boundaries by either (1) locating the start and end of the total magnetic field enhancement relative to the background field strength as the beginning and end of the flux rope, (2) using the minima associated with the core field component enhancement, or (3) using the maxima of the bipolar field. After boundary identification, we applied minimum variance analysis (MVA) to the SE, GSE, or RTN coordinates in which the maximum coordinate was the field rotation and the intermediate coordinate was the axial core field [Lepping et al., 1990]. Our standards were such that the maximum eigenvector $\left(\lambda_{1}\right)$ divided by the minimum eigenvector $\left(\lambda_{3}\right)$ must be equal to or greater than six and the intermediate eigenvector $\left(\lambda_{2}\right)$ divided by the minimum eigenvector $\left(\lambda_{3}\right)$ must be equal to or greater than two, which will guarantee well determined MVA directions [Sonnerup and Cahill, 1967; Burlaga and Behannon, 1982]. We excluded events if the bipolar field was primarily in the $B_{X} \mathrm{GSE} ; B_{R} \mathrm{RTN} ; B_{X}$ $\mathrm{SE}$ coordinate as to remove any potential heliospheric current sheet crossings. However, we include events that had $B_{X}$ crossings if the bipolar field was primarily in one of the other two coordinates (as indicated by the maximum eigenvector).

[19] The second step in our selection process was the removal of compressive Alfvén wave-like events; at these small scales, there are several disturbances that can bias surveys unless taken into account. As described in section 1., the magnetic field and velocity components were examined to see if they were correlated. If the correlation coefficient was $\geq 0.7$, suggestive of Alfvénic waves, we excluded the event from the flux rope database. From the initial database of the combined ACE, Wind, IMP 8, and Ulysses events, one third of candidate small-scale flux ropes had Alfvénic properties and were removed from the final database. Those events will be the subject of a future study, but their relative high occurrence indicates that they should be carefully excluded in studies of flux ropes. The inclusion of these events has led to differences between data sets of solar wind flux ropes.

[20] The events were then validated using the nonlinear least squares method. The data were fitted to the force-free, cylindrically symmetric flux rope model discussed in the introduction. We used the same method as described in Lepping et al. [1990]. In this study, we did not use the assumption that the spacecraft passes through the center of the flux rope but used the nonlinear, least-squares minimization of the data to the force-free, cylindrically symmetric flux rope model using five free parameters: the flux rope longitude $(\varphi)$ and latitude $(\theta)$, the impact parameter $(p)$, axial field strength $\left(B_{o}\right)$, and the sign of the helicity $(H)$. The $\chi_{D I R}^{2}$ error from the model fit of the flux rope axis is used to evaluate the goodness of fit, where an error $>0.3$ is typically a poor fit [Lepping et al., 1990]. We labeled events as flux ropes if the error was up to 0.5 . The flux rope axis can have any orientation $\left(\phi_{\text {Rope }}, \theta_{\text {Rope }}\right)$ relative to the ecliptic plane. The observed directions of the magnetic field at the leading edge, center, and trailing edge of the flux rope are commonly used to classify magnetic clouds [Bothmer and Rust, 1997; Bothmer and Schwenn, 1998; Mulligan et al., 1998]. There are two broad classifications, the first is where the flux rope axis lies in the ecliptic plane (e.g., in the $B_{Y}$ GSE component) and the second where the axis lies out of the ecliptic plane (e.g., in the $B_{Z}$ GSE component). We have classified our events in the same manner. In this study, we are interested in the basic properties of the flux ropes; their orientation; duration of the event, which is slightly modified from the original visual boundaries because of the leastsquares minimization technique; and the strength of the axial field extracted from the model.

\section{Results}

\subsection{Examples of Small-Scale Flux Ropes at Various Radial Heliospheric Distances}

[21] We identified 23 small-scale flux ropes from the Helios 1 and Helios 2 data sets, 66 flux ropes from the IMP 8 data set, 91 flux ropes from the Wind data set, 70 flux ropes from the ACE data set, and 20 flux ropes from the Ulysses data set. There were 51 events identified in both the Wind and ACE databases and 12 events found in the ACE data set but not in the Wind data set. We excluded the unique ACE events and created a combined $1 \mathrm{AU}$ database using the Wind and ACE events that contains a total of 98 unique flux ropes. There are a total of 219 unique smallscale flux ropes at radial distances from 0.3 to $5.5 \mathrm{AU}$. The flux ropes range in duration from 0.22 to $10.35 \mathrm{~h}$. There are several flux ropes in our database that have been previously identified [Feng et al., 2007, 2008; Cartwright and Moldwin, 2008; Jian et al., 2008]. The Helios 1 and Helios 2, IMP 8, 

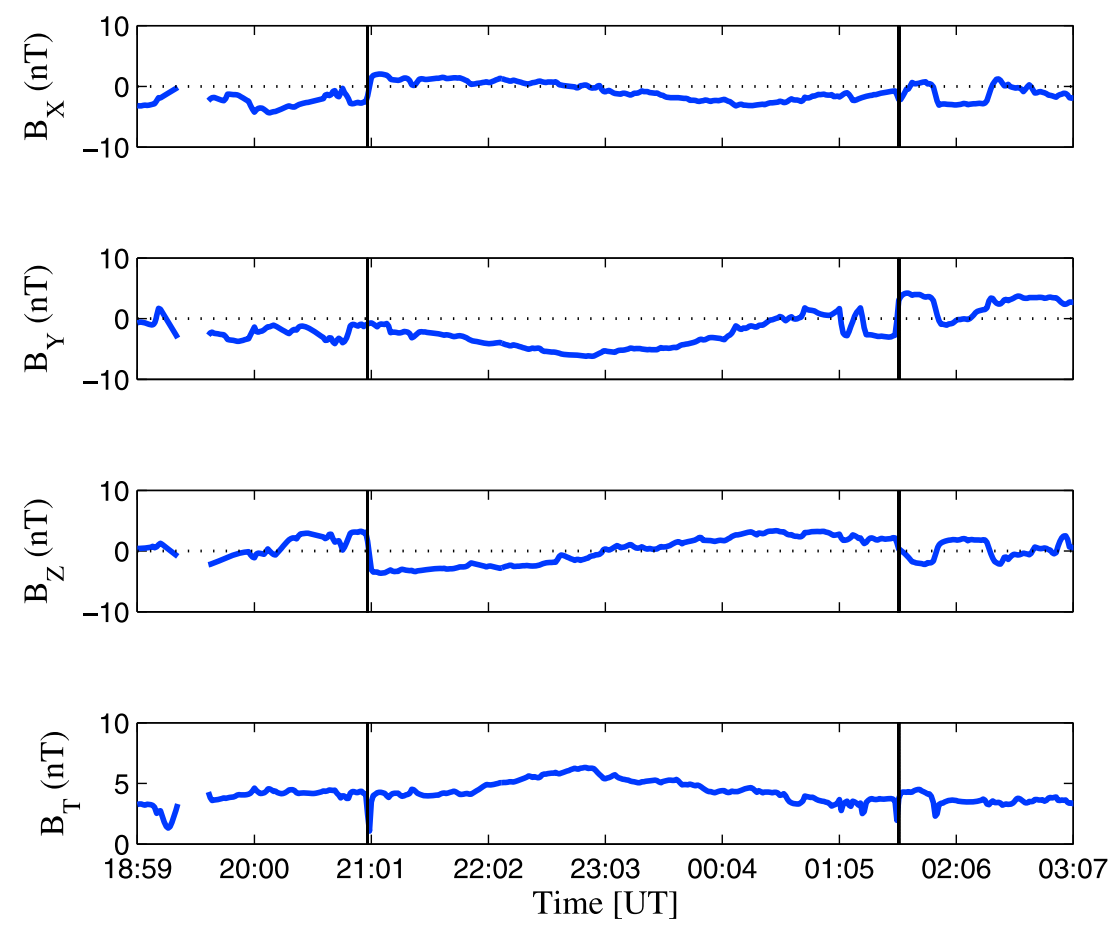

Figure 2. A classic example of a small-scale flux rope located at $1 \mathrm{AU}$. It was identified from the Wind data set from 11 August 1996 at 2059 to 12 August 19960136 UT. The boundaries are represented by the solid vertical lines.

and ACE/Wind combined database and Ulysses small-scale flux rope database can be found in Appendix A, Table A1.

[22] We present three examples at $0.3,1$, and 5 AU radial distances. The first flux rope presented in Figure 1 was observed by the Helios 1 spacecraft on 4 March 1975 from 02:19 to 02:47 UT. The axial core field strength was $35 \mathrm{nT}$. The duration of the event was $0.47 \mathrm{~h}$. It was observed at a heliospheric distance of $0.39 \mathrm{AU}$. The hourly plasma data indicate it was located in the fast solar wind with a speed of $630 \mathrm{~km} \mathrm{~s}^{-1}$. The $\lambda_{1} / \lambda_{3}$ ratio was 21 and the $\lambda_{2} / \lambda_{3}$ ratio was 16. The ratio of the axial field strength to the average $6 \mathrm{~h}$ surrounding the flux rope was 1.8. The orientation of the flux rope was such that the leading field from the bipolar signature is in the south direction and the trailing field is in the north direction. The axial field is in the west direction, so the event is an SWN event. The flux rope is a right-handed event and is embedded in the away interplanetary magnetic field (IMF) polarity sector. The longitude of the axis is $280^{\circ}$ and the latitude is $-25^{\circ}$, with respect to the ecliptic plane.

[23] The second example presented in Figure 2 was identified with the Wind spacecraft starting on 11 August 1996 at 20:59 UT to 12 August 1996 at 01:36 UT. The duration of the event was $4.62 \mathrm{~h}$. The axial field strength was $5.34 \mathrm{nT}$. The $\lambda_{1} / \lambda_{3}$ ratio was 50.7 and the $\lambda_{2} / \lambda_{3}$ ratio was 12.3 . The average solar wind speed over the duration of the event was $350 \mathrm{~km} \mathrm{~s}^{-1}$. The latitude and longitude of the axis is $76^{\circ}$ and $-17^{\circ}$. The flux rope is again classified as an SWN event with a right handedness.

[24] The third example was found by the Ulysses spacecraft at 5.3 AU from 28 November 2004 at 06:05-07:55 UT and is presented in Figure 3. The axial field strength is $0.74 \mathrm{nT}$ and the axial field strength divided by the surrounding $6 \mathrm{~h}$ field strength is 1.07 . The duration is $1.83 \mathrm{~h}$.
The average solar wind speed over the duration of the event was $427 \mathrm{~km} \mathrm{~s}^{-1}$. The $\lambda_{1} / \lambda_{3}$ ratio was 156 and the $\lambda_{2} / \lambda_{3}$ ratio was 7 . The flux rope longitude and latitude are $77^{\circ}$ and $-3^{\circ}$. The flux rope is orientated such that it is SEN event with a left handedness.

\subsection{Basic Properties at $1 \mathrm{AU}$ and Heliospheric Evolution Statistics of Small-Scale Flux Ropes}

[25] We used the Helios 1, Helios 2, IMP 8, Wind/ACE, and Ulysses small-scale flux rope database to study the number of events observed per year and how their properties depend on heliospheric distance. How frequently smallscale flux ropes are observed is a fundamental property that gives clues to their possible origin. The occurrence of smallscale flux ropes over solar cycles 21,22 , and 23 is presented in the upper plot of Figure 4. We plotted the number of events normalized by number of spacecraft months of observation per year represented as black diamonds. We used this normalization because of the deviations in spacecraft coverage from the various data sets used. The error bars are the plus and minus square root of (the number of events) divided by the months of observations per year. The solid gray line connecting the solid circles is the mean annual sunspot number per year. This plot shows a trend of small-scale flux rope occurrence, with the events most likely observed during solar minimum than solar maximum. We used these observations to create the average number of flux ropes per month of observation for cycles 21 to 23 as a function of solar cycle, shown in the lower plot of Figure 4. This analysis summed over observations at the same phase of the solar cycle in each cycle (e.g., $t, t+11$, and $t+22$ ), and then we plotted the average rate (black diamonds). For example, the first bin is the average number of flux ropes for 

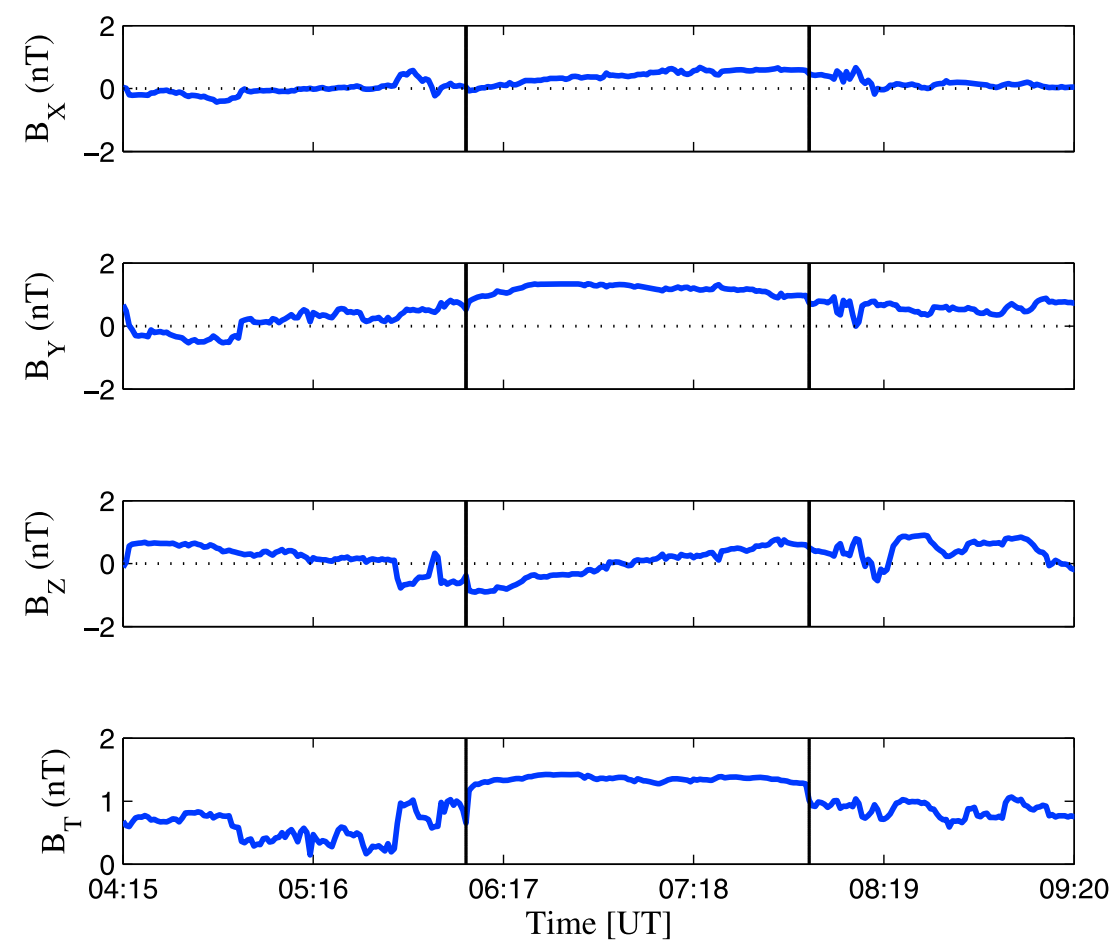

Figure 3. An example of a small-scale flux rope located at 5.3 AU. This event was identified from the Ulysses data set starting on 28 November 2004 at 0605 to 0755 UT. The boundaries are represented by the solid vertical lines.

years $1974,1974+11$, and $1974+22$ divided by the average number of months of observation for those years. The error bars are the (average number of flux ropes) divided the average months of observation. The sunspot number (solid gray line) is the average number of sunspots for that year from the cycles 21 to 23. This plot does not include the year 2007 because the Sun started going into an extended minimum and it does not fit with the previous two cycles. This plot shows the same trend as the upper plot, where there are more small-scale flux ropes observed during solar minimum than maximum. The average number of events around solar minimum (taken as years 1, 2, 10, and 11 in the cycle) is one flux rope per month. The average number of events around solar maximum (here taken as years 5 through 7) is 0.4 flux ropes per month. This average trend shows that there is a tendency to observe more smallscale flux ropes during solar minimum than maximum.

[26] The number of small-scale flux ropes normalized by months of observation as a function of radial heliospheric distance is presented in Figure 5. The error bars are the square root of the (number of events) divided by the months of observation. The first column in Table 1 specifies the four regions examined: the inner heliosphere (0.3-0.75 AU), near $1 \mathrm{AU}(0.75-1.5 \mathrm{AU})$ where we have the most months of observations, and then the outer heliosphere, which we have separated into 1.5-4.5 and 4.5-5.5 AU. There are not many events in the 1.5-4.5 AU bin because of lack of spacecraft observing hours, but the events follow the same trend as the 4.5-5.5 AU bin. In the inner heliosphere, the number of events per month is 0.8 , which declines to 0.6 at $1 \mathrm{AU}$ and further declines to 0.4 in the outer heliosphere (5 AU). The dashed black line is the least squares fit of the function $\left(R^{-x}\right)$ to the data, where $x$ indicates how fast the distribution falls off with heliospheric distance. In this case, the distribution falls as $R^{-0.24}$, indicating the number of flux ropes as a function of heliospheric distance is not flat. There is a small decrease in the occurrence frequency as a function of radial distance with more events in the inner heliosphere (0.3-1 AU) compared to the outer heliosphere (5 AU).

[27] The duration and scale size of small-scale events as a function of heliospheric distance are presented in Figure 6. The upper plot shows the average flux rope duration per heliospheric bin indicated by the black diamonds and the individual flux rope durations that created the average are plotted in gray. In the inner heliosphere, the average duration is $1.5 \mathrm{~h}$. The average duration is $2.5 \mathrm{~h}$ at $1 \mathrm{AU}$ and flattens to 4 to $4.3 \mathrm{~h}$ in the outer heliosphere. The dashed black line is the least squares fit of the function $\left(R^{x}\right)$ to the average duration data; this indicates how the rate at which the duration increases as a function of heliospheric distance. The fit to the data is $R^{0.37}$, indicating the duration in the inner heliosphere has a large increase and then increases more slowly with increasing heliocentric distance. The lower plot shows the average scale size (in units in AU) as a function of heliospheric bin, plotted as the black diamonds. The average flux rope scale size in the inner heliosphere $(<0.75 \mathrm{AU})$ is $0.0132 \mathrm{AU}\left(311 R_{E}\right)$, at $1 \mathrm{AU}$ it is $0.0247 \mathrm{AU}$ $\left(582 R_{E}\right)$, and in the outer heliosphere (5 AU) it is 0.0383 AU $\left(900 R_{E}\right)$. The dashed black line is the least squares fit of the average data to the function $R^{x}$. The fit is found to be $R^{0.43}$. These plots show a trend where an average event in the inner heliosphere is smaller in duration and scale size than an average event at $1 \mathrm{AU}$ and beyond. 

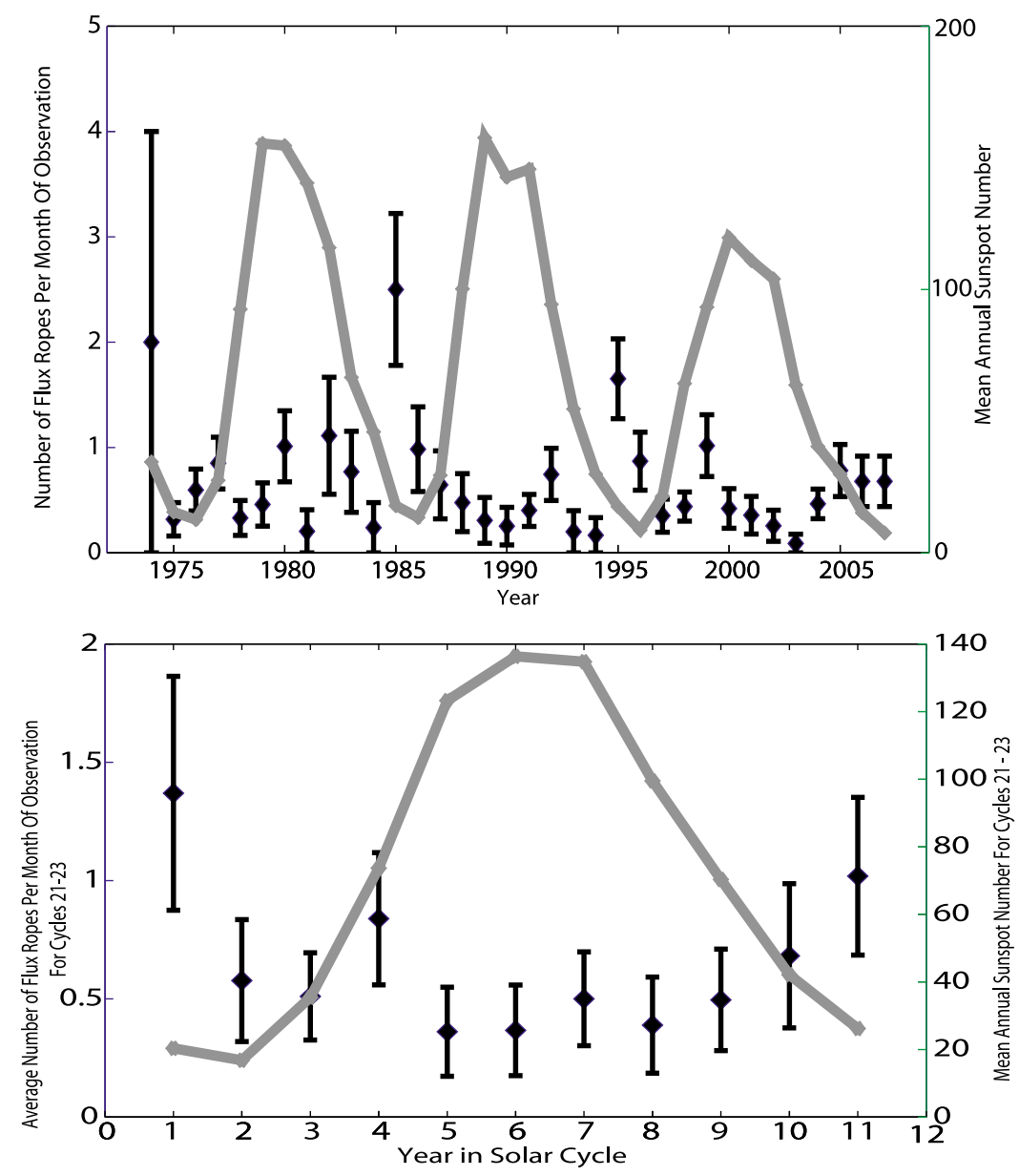

Figure 4. The upper plot is the number of small-scale flux ropes normalized by the months of observations per year from 1974 to 2007, covering solar cycles 21 to 23 . The diamonds are representative of the number of events per month of observation per year. The error bars are the square root of the (number of events) divided by the number of months of observation per bin. The solid gray line represents the mean annual sunspot number. The bottom plot is the average number of small-scale flux ropes per months of observation over solar cycles 21 to 23 .

[28] One of the parameters of the cylindrically symmetric model fit was the axial core field strength. This parameter was extracted and binned as described in Table 1. The average axial core field strength of the small-scale flux ropes as a function of heliospheric distance is presented in the upper plot of Figure 7. The error bars are plus and minus the standard deviation of the average axial core flux rope field strength divided by the square root of the number of events. In the inner heliosphere, the first bin, the average axial core field was $17 \mathrm{nT}$, rapidly decreased to $7 \mathrm{nT}$ at $1 \mathrm{AU}$, and then flattened to approximately $2 \mathrm{nT}$ in the outer heliosphere. The black dashed line is the least squares fit of the data to the function $\left(R^{-x}\right)$, where the result found is $R^{-0.94}$. The ratio of the axial core field strength to the surrounding background interplanetary field strength determines how strong the small-scale flux rope is compared to the IMF it resides in. The average ratio of axial core field strength to background solar wind IMF as a function of heliospheric distance is present in the lower panel of Figure 7. The ratio is slightly larger in the inner heliosphere at 1.3 and is flat from 1 to $5 \mathrm{AU}$ at around 1.1. The least squares fit of the data to the function $\left(R^{-x}\right)$ is found to be $R^{-0.07}$. This distribution and the error bar size between the data points indicate the strength of small-scale flux ropes relative to the background solar wind is roughly constant from the inner to outer heliosphere.

[29] We examined the plasma behavior of small-scale flux ropes at $1 \mathrm{AU}$ with the database of events identified with the ACE spacecraft, of which there were 70 events identified from 1998 to 2007. To understand the cumulative plasma behavior before, during, and after the event, we analyzed the events with a normalized superposed epoch analysis. The zero epoch time was defined as the inflection point of the flux rope bipolar field, found in either $B_{Y}$ or $B_{Z}$ GSE coordinates. We flipped the bipolar rotation such that the rotation of the bipolar field was from the negative sector to the positive sector. This helped to draw the eye to the beginning, inflection point, and end boundary of the flux rope. The duration distribution of the events is scattered from $\sim 1 \mathrm{~h}$ up to $8 \mathrm{~h}$, with the mean duration on the $\sim 2 \mathrm{~h}$. To understand the cumulative behavior of these events, we normalized the duration of each event to an approximate mean duration of $2 \mathrm{~h}, 1 \mathrm{~h}$ before the inflection point and $1 \mathrm{~h}$ after the inflection point. In addition to this normalization, 


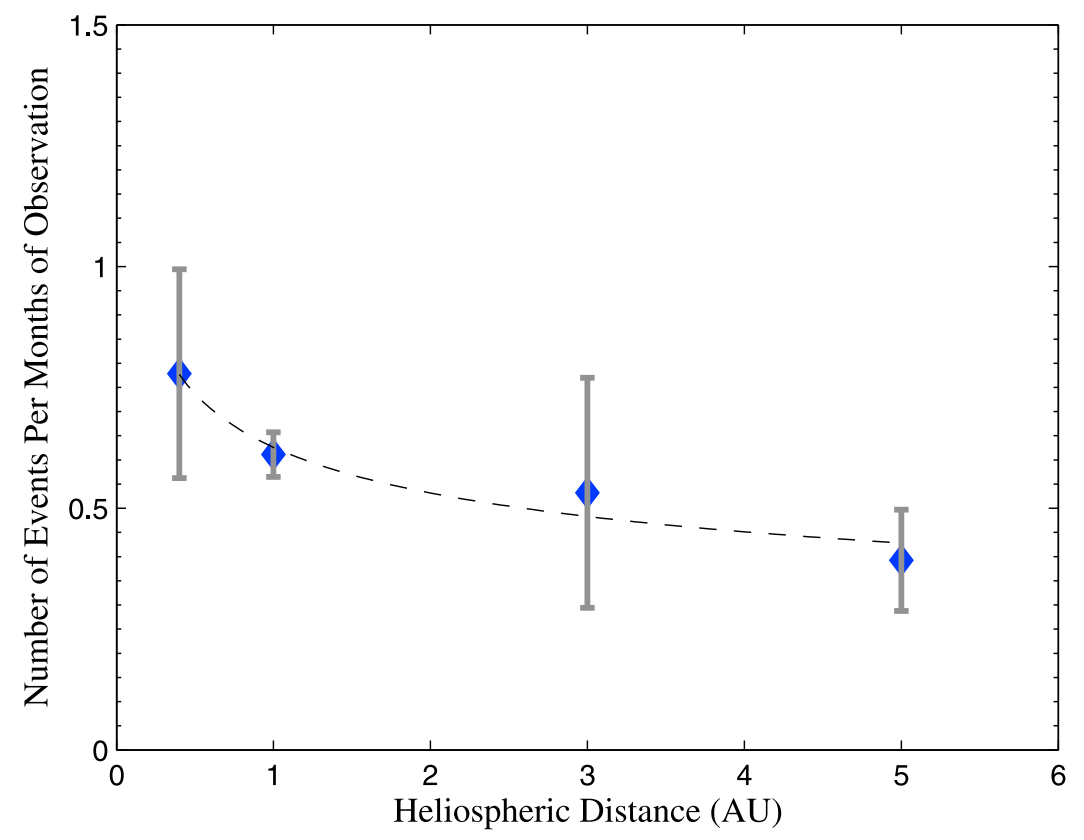

Figure 5. The number of small-scale flux ropes normalized by month of observation as a function of the radial heliospheric distance. The error bars are the square root of (the number of events) divided by the months of observation per bin. Table 1 specifies the number of months of observation per bin. The dashed line is the least squares fit, where the radial dependence is $R^{-0.24}$.

we found that in order to not have a sharp discontinuity between the boundaries of the cumulative events and the surrounding solar wind magnetic and plasma data we overlapped the normalization with part of the surrounding field and plasma data ( $\sim 30 \mathrm{~min}$ on each side of the event). After we constructed the matrix of normalized events with the surrounding magnetic and plasma data, the upper, median, and lower quartiles were determined. The superposed epoch is presented in Figure 8. The upper level is the $75 \%$ quartile and is represented as the upper red line, the median value is the $50 \%$ quartile (the middle blue line), and the lower level is the $25 \%$ quartile (the lower red line). The first panel is the bipolar field of the flux rope, which is either in the $B_{Y}$ or $B_{Z}$ GSE direction. The second panel is the total magnetic field strength. The third panel is the total velocity in $\mathrm{km} \mathrm{s}^{-1}$. The total velocity is constant across the inflection point and is consistent with predominately slow solar wind $\left(<410 \mathrm{~km} \mathrm{~s}^{-1}\right)$. The number density of protons is the fourth panel and shows a constant behavior across the inflection point. The density upper quartile shows a slight enhancement before the small-scale flux rope event, similar to the sheath region formed by swept up ambient solar wind magnetic clouds often display [Lepping et al., 2006]. Beta is the ratio of plasma pressure to magnetic pressure, plotted in the fifth panel and begins to weaken at the start of the smallscale flux rope with the strongest depression at the inflection point. The total pressure is plotted in the sixth panel and is defined as the sum of the proton plasma pressure and the magnetic pressure $\left(P_{T}=n_{P} k T_{P}+B^{2} / 2 \mu_{0}\right)$. It shows an extremely weak enhancement in the median of $0.03 \mathrm{nPa}$ at the inflection point. The temperature of protons $(\mathrm{K})$ is the last plot and shows a constant profile across the inflection point.
[30] We investigated the proximity of the Wind database of events to the nearest sector crossing (e.g., heliospheric current sheet crossing). The $B_{\varphi}$ GSE vector is defined as starting from the Earth to the Sun, rotating counterclockwise, such that the "away" sector was at a longitude of $135^{\circ}$ and the "toward" sector was $315^{\circ}$. Sector crossings were visually identified in hourly $B_{\varphi}$ GSE vector data as a $\sim 180$ degree rotation from one sector polarity to the other. Rotations from one polarity to the other had to remain "stable" which we defined as longer than a day (e.g., if the $B_{\varphi}$ vector rotated from one polarity to the other and then rotated back to the original polarity within a day we did not count this as a sector crossing) [Lepping et al., 1996]. This was done to remove random fluctuations and ICMEs, which can disturb the heliospheric current sheet in such a manner. Sector crossings near small-scale flux ropes that were very complex and had no sustained polarity change were discarded; there were 20 events removed for this reason, leaving 71 events in the distribution. The distribution of the small-scale flux rope start time to the nearest sector crossing is presented in Figure 9. The time to sector crossing is binned is every 0.25 days. There is a very sharp peak with 17 events observed within 0.25 days of a sector crossing. The distribution has a sharp peak and is skewed with a preference before the sector crossing (skew-

Table 1. Number of Small-Scale Flux Ropes per Location and Months of Observation

\begin{tabular}{ccc}
\hline Heliospheric Distance (AU) & Number of Events & Months of Observation \\
\hline$<0.75$ & 13 & 16.7 \\
$0.75-1.5$ & 175 & 286.3 \\
$1.5-4.5$ & 5 & 9.4 \\
$4.5-5.5$ & 14 & 35.7 \\
\hline
\end{tabular}



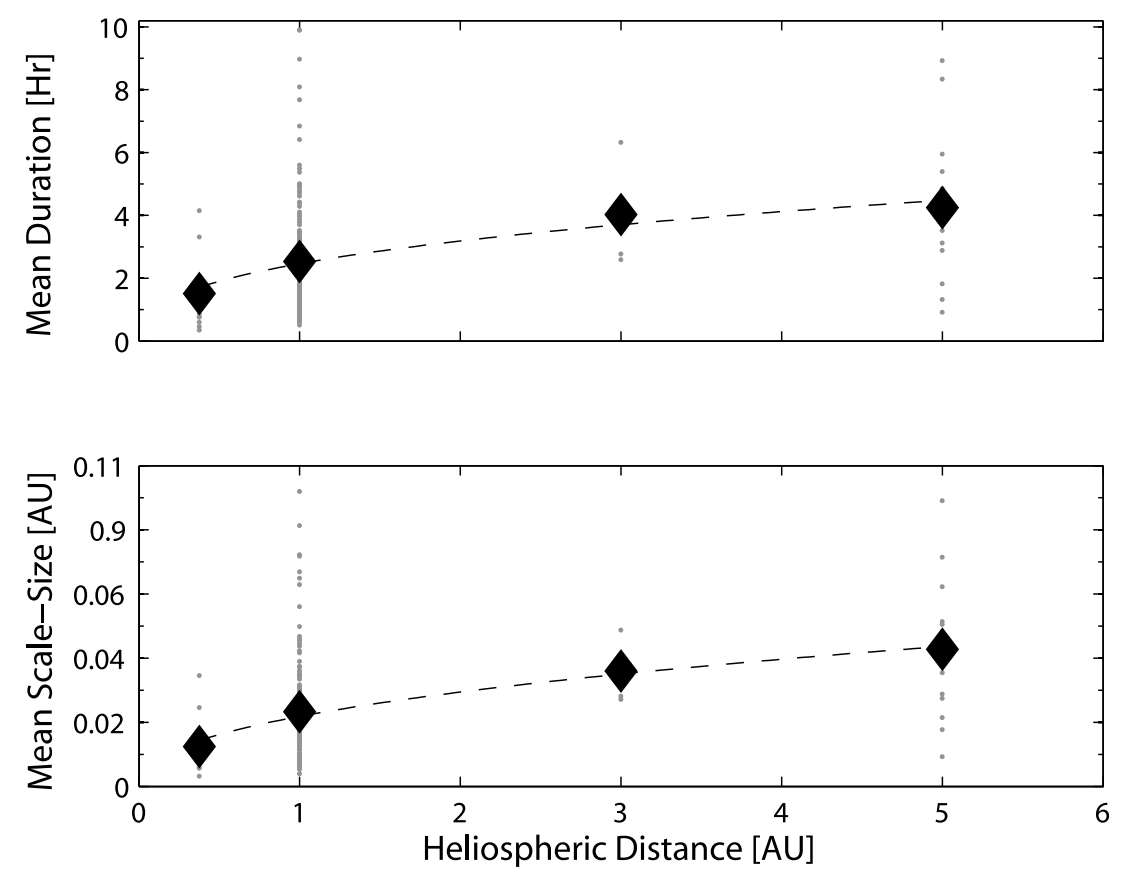

Figure 6. The top plot is the average small-scale flux rope duration per heliospheric distance binned according to Table 1, shown in black diamonds. The dashed line is the least squares fit to the data, where the radial dependence is $R^{0.37}$. The bottom plot is the average scale size of each flux rope as a function of heliospheric distance. The dashed line is the least squares fit, where the dependence is $R^{0.43}$. Each small-scale flux rope, binned per heliospheric distance, duration, and scale size, respectively, is plotted as a gray point.

ness is -0.22 ). The distribution has a mean of 0.016 days $\pm \mathrm{a}$ standard deviation of 1.4 days.

\section{Discussion}

[31] There is a debate as to where small-scale flux ropes originate: (1) they are formed through magnetic reconnection in the solar wind such as across the heliospheric current sheet [Moldwin et al., 1995, 2000; Cartwright and Moldwin, 2008], (2) they form in the corona similar to MCs [Feng et al., $2007,2008]$, or (3) there are two populations, one originating in the corona and the other in the solar wind. The evolution of small-scale flux rope properties found here can help address this debate. The first suggestion that they are created in the solar wind through reconnection [Moldwin et al., 1995, 2000] was made because (1) the scale sizes of small-scale flux ropes are much smaller than MCs, (2) they lack a depressed proton temperature like $\mathrm{MCs}$, and (3) they lack an expansion signature at 1 and 5.3 AU.

[32] The second hypothesis was put forth by Feng et al. [2007, 2008], whose studies suggest that these objects are small-scale versions of MCs with a solar origin. The reasoning was from the results of these studies at $1 \mathrm{AU}[$ Feng et al., 2007, 2008] using the spacecraft Wind over solar cycle 23. These results showed that small-scale flux ropes and MCs do not have strong dependence on solar cycle, both are predominately in the slow wind, have stronger core field strength as compared to the background solar wind, and their axial longitudes and latitudes are similar [Feng et al., 2008]. The studies by Feng et al. [2007, 2008] did not remove objects from the database that were Alfvénic.
This hypothesis is further supported by observations from Mandrini et al. [2005], who identified a sigmoid on the Sun and correlated it to a small-scale flux rope in the solar wind. This was done through timing the eruption and subsequent observation in the solar wind and comparison of the helicity of the two objects [Mandrini et al., 2005]. This event was not included in our database because it was identified as an Alfvén wave. This hypothesis also does not explain why the proton temperature is not depressed in small-scale flux ropes as it is for MCs. The results from this study will help to shed light on these origin theories.

[33] Our results indicate the occurrence rate of small-scale flux ropes per year has a weak anticorrelation dependence on solar cycle. This result is consistent over three solar cycles (21-23), where on average there is 1 small-scale flux rope per month around solar minimum and 0.4 events per month around solar maximum. In comparison with largescale flux ropes (MCs), their occurrence rate is not as dependent on the phase of the solar cycle. However, the study of ICMEs by Richardson and Cane [2004] found the fraction of MCs in the ICME database at solar minimum to be $\sim 100 \%$ and at solar maximum to be $\sim 15 \%$ during solar cycle 23 . They also found that this trend was consistent in solar cycles 21 and 22. It is unclear why MCs are not as prevalent at solar maximum given the rate of ICMEs greatly increases. There have been suggestions on why MCs are less observed compared to ICMEs during solar maximum; these ideas might be related to why small-scale flux ropes are observed less during solar maximum.

[34] There are two views of why flux ropes are preferentially observed during solar minimum over ICMEs. As 

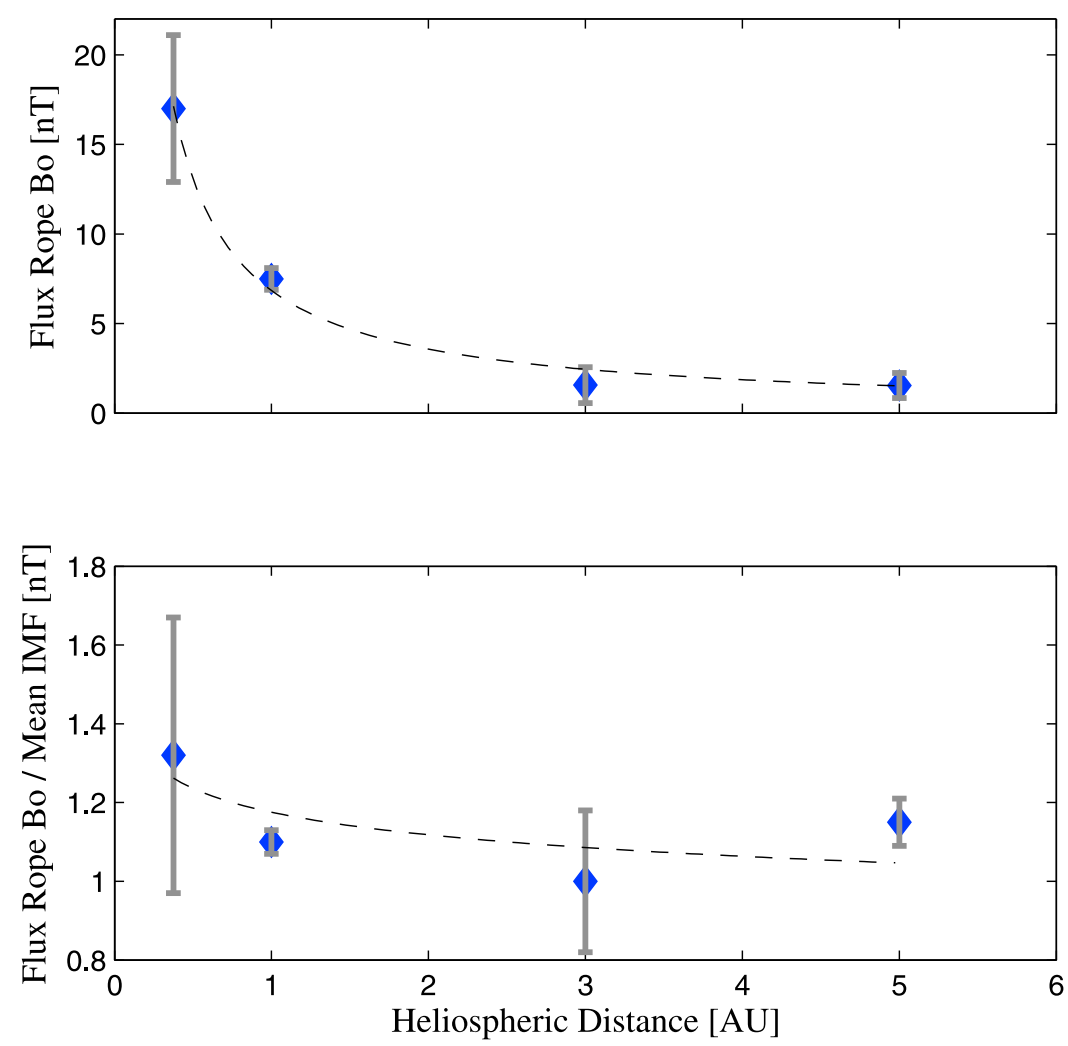

Figure 7. The top plot is the axial field strength of small-scale flux ropes versus heliospheric distance. The dashed line is the least squares fit to the data, where the radial dependence is $R^{-0.94}$. The lower plot is the axial field strength divided by the average IMF field strength of the surrounding $6 \mathrm{~h}$, with the binning shown in Table 1. The dashed line is the least squares fit to the data, where the radial dependence is $R^{-0.07}$. The error bars are plus or minus the reduced error, which is the standard deviation of the average flux rope strength divided by the square root of the number of events.
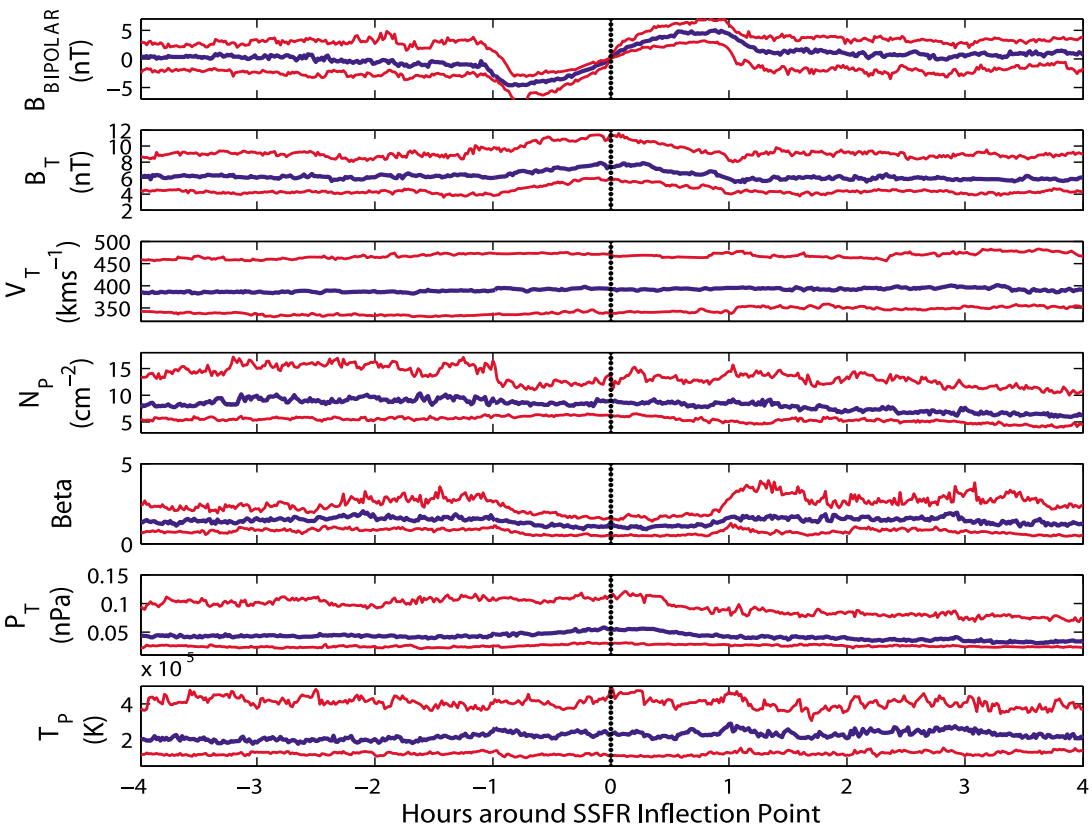

Figure 8. Superposed epoch analysis of the plasma properties of small-scale flux ropes at 1 AU. 


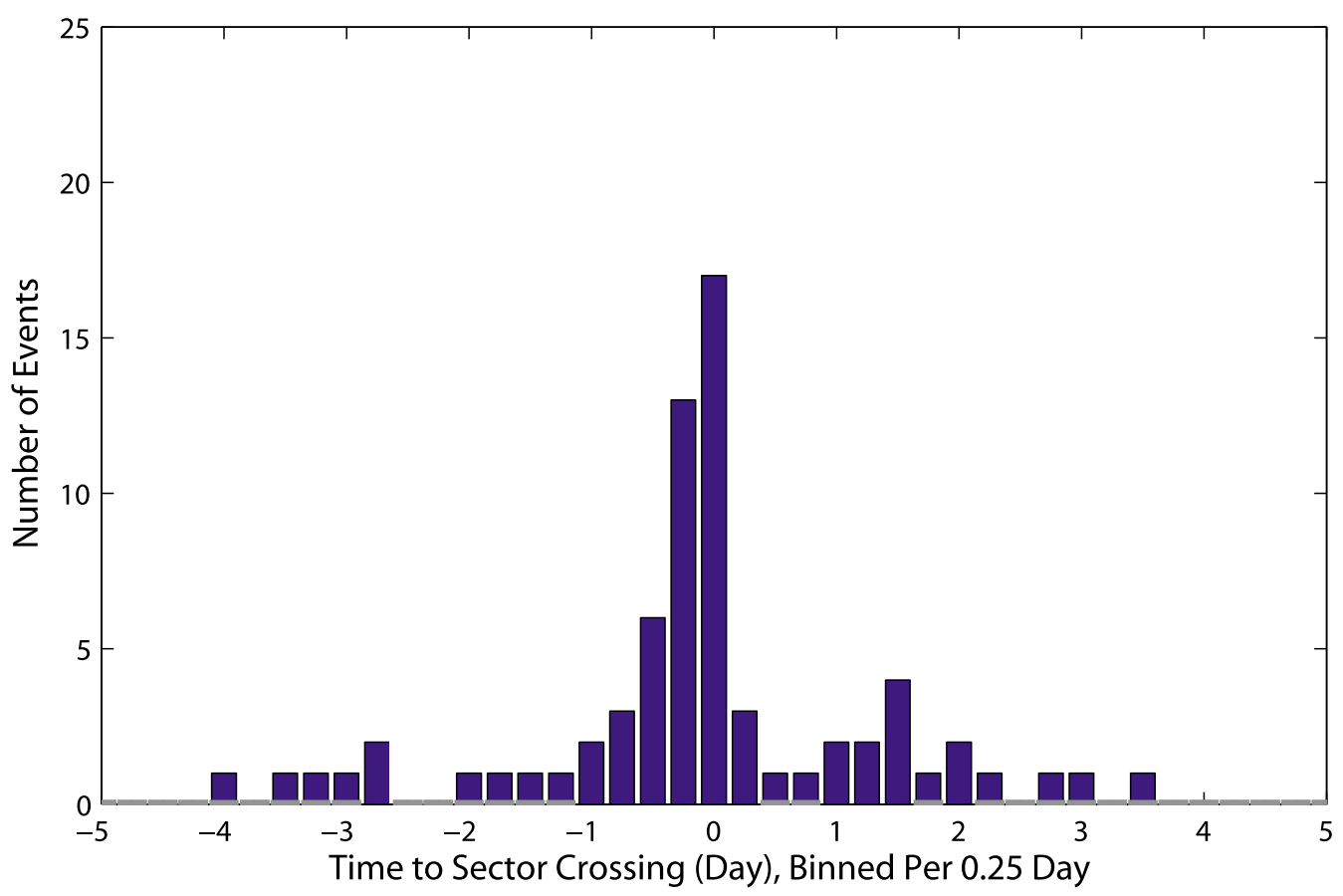

Figure 9. The distribution of events as a function of the time difference between a sector boundary crossing and a small-scale flux rope. The time to sector crossing is binned every 0.25 days, where the negative values indicate the flux rope preceded the sector crossing.

solar activity increases, the latitudinal extent of a CME core axis also increases. This term is called the apparent latitude [Yashiro et al., 2004]. This could mean the magnetic clouds will only make a glancing encounter with Earth. The study by Henke et al. [2001] showed with Ulysses data that the percentage with high latitudes ICMEs to low latitude ICMEs increased over solar maximum. It is not clear if this is a true latitudinal change or if this is just consistent with enhanced ICME activity during solar maximum. The second view is that during solar minimum the simple flux rope configuration is more common than during solar maximum where magnetic complexity increases. Mulligan et al. [1998] showed higher inclination magnetic clouds are more likely to occur at solar maximum than solar minimum. There are reports of multiple flux ropes rotations within ICMEs during solar maximum [ $W u$ and Lepping, 2007]. The results of this study are consistent with the view that as solar magnetic field complexity increases the occurrence of simple flux rope structure decreases. If small-scale flux ropes are being created at the Sun, then these simple force-free structures are observed more easily during solar minimum consistent with the decrease in magnetic complexity.

[35] The average number of events in the inner heliosphere is slightly greater than the outer heliosphere as seen from Figure 5. The distribution as a function of distance declines as $R^{-0.24}$. This distribution indicates the majority of events lies between 0.3 and $1 \mathrm{AU}$ and then begins to declines between 1 and 5 AU. This implies the source of these small-scale flux ropes is in the inner heliosphere. This trend is more compelling when combined with the result from Figure 6 showing the increase in duration (and scale size) from the inner to outer heliosphere. The increase in duration from 0.3 to $1 \mathrm{AU}$ is rapid and after $1 \mathrm{AU}$ begins to flatten out. These observations suggest that small-scale flux ropes are rapidly expanding from their source in the inner heliosphere and their expansion rate slows between 1 and 5 AU.

[36] These results indicate the characteristics of smallscale flux ropes evolve as a function of heliospheric distance. As discussed in the previous paragraph, Figure 6 shows the average duration and scale size of small-scale flux ropes as a function of radial distance. The plot indicates a large, rapid expansion within $1 \mathrm{AU}$ and then a much more gradual change with radial distance. The average rate of scale-size expansion varies with heliospheric distance as a function of $R^{0.43}$. This rate of expansion is weaker than the radial dependence of ICMEs, which continue to expand well past $1 \mathrm{AU}$ and whose rate of expansion varies from $R^{0.5}$ to $R^{0.9}$ [Forsyth et al., 2006]. The average axial core field strength of small-scale flux ropes drops off as $R^{-0.94}$ with heliospheric distance, as shown in Figure 7. This is slower than what is observed for the mean magnetic field of ICMEs, which drops as $R^{-1.3}$ to $R^{-1.5}$, as discussed in Forsyth et al. [2006]. Although these two parameters are not exactly the same, it is useful for comparison purposes to understand that both small-scale flux ropes and ICMEs magnetic field strength decline with radial distance. The ratio of the core field strength to the background IMF field is constant (within error bars) from the inner to outer heliosphere. The ratio is slightly greater than 1, implying smallscale flux ropes are slightly stronger than the background IMF. This suggests that these events survive out to $5.5 \mathrm{AU}$ after being formed in the inner heliosphere or that they are being formed at various heliospheric distances.

[37] The superposed epoch analysis shows that smallscale flux ropes have finished their evolution at $1 \mathrm{AU}$ because they are force-free, cylindrically symmetric magnetic field structures with a constant velocity profile. This is consistent with previous observations of the structure of flux 
ropes at 1 AU [Moldwin et al., 2000; Feng et al., 2007 2008; Cartwright and Moldwin, 2008]. The proton temperature is constant across the duration of the event, where for magnetic clouds there is a temperature depression at 1 AU. The median of the proton density is constant across the duration of the event, giving further evidence to support the forcefree nature of these events at $1 \mathrm{AU}$. The upper quartile of the proton density does show an enhancement before the event starts. This shows that there are some events that move slightly faster than the background plasma and sweep up some of the plasma in front of it creating a sheath region. This is commonly observed at the beginning of magnetic clouds. The overall behavior is best represented by the median density that remains constant across the event. The plasma beta decreases over the duration of the flux rope due to the enhancement in the total field. The total pressure shows an enhancement at the middle of the event. Since the density and temperature remain constant, the enhancement comes from the axial core field of the flux rope, which peaks at the center of the event. This total pressure peak at the center also gives further evidence of the force-free nature of these events.

[38] We investigated the solar wind context of small-scale flux ropes. We presented the time to the nearest sector crossing from the center of the small-scale flux rope in Figure 9. The results indicate that small-scale flux ropes are most often located near sector crossing ( $\sim 6 \mathrm{~h}$ or less) and are more likely to be before the sector crossing. There are a few events found inside an ICME sheath region and the ICME itself, where the ICMEs identification is from the databases of Jian et al. [2008] and Cane and Richardson [2003]. Although we removed events that displayed compressive Alfvén wave characteristics with a correlation of 0.7 , there were still approximately $10 \%$ of events that were in regions of solar wind with high shear Alfvén activity. It is possible these events are compressive Alfvén waves rather than flux ropes and could bias our results; we are working on a future study investigating these waves.

\section{Conclusions}

[39] This is the first investigation that observed smallscale flux ropes at a range of heliospheric radial distances, from 0.3 to $5.5 \mathrm{AU}$. We found that small-scale flux ropes are most likely to occur during solar minimum rather that solar maximum. This trend is consistent over solar cycles 21 to 23. This implies their creation mechanism is dependent on solar cycle. Their properties (occurrence, duration, and axial field strength) evolve from the inner to outer heliosphere. When combining their properties with the occurrence as a function of radial heliospheric distance, the results have shown that small-scale flux ropes are created within 1 AU. The duration of events as a function of heliospheric distance implies that there is a rapid expansion within $1 \mathrm{AU}$ and they remain relatively stable to $5 \mathrm{AU}$, consistent with the events formed within 1 AU. They are most likely observed near the sector crossing.

[40] The hypothesis that small-scale flux ropes form in the corona similar to MCs [Feng et al., 2007, 2008] is supported by the dependence on solar cycle and the expansion of the scale size from the inner to outer heliosphere (similar to MCs). However, if small-scale flux ropes were ejected off the Sun like magnetic clouds, then they should also have similar plasma signatures. Why do they lose their plasma signature by the time they reach 1 AU? Our results also indicate small-scale flux ropes are observed near the sector boundary crossings. This solar origin hypothesis would allow for events to be ejected from any latitude and does not explain the preference of the events to be found near the sector boundary. CMEs are regularly observed by the Large Angle Spectroscopic Coronagraph (LASCO) experiment on the SOHO satellite [Brueckner et al., 1995]. If small-scale flux ropes are small magnetic clouds, it is possible to image the very largest of them being ejected off the Sun using the $\mathrm{C} 1$ coronagraph, but the $\mathrm{C} 1$ coronagraph failed early in the SOHO mission. We can extrapolate the size of small-scale flux ropes from the inner heliosphere to the Sun using the information from Figure 6. If we assume to the solar wind was on average $400 \mathrm{~km} \mathrm{~s}^{-1}$, consistent with observations for small-scale flux ropes [Cartwright and Moldwin, 2008], and do a simple linear extrapolation of the expansion rate back to the Sun, then the scale size in the corona is between $\sim 1$ and $400 \mathrm{Mm}$. This is near the edge of the resolution range of LASCO C2 (17 Mm) and C3 (87 Mm) [Brueckner et al., 1995]. However, the properties of small-scale flux ropes included in this study have different plasma and evolutionary properties from MCs and hence are consistent with a different formation mechanism suggestive that they are not just the small-sized tail of MCs.

[41] The hypothesis that they are created due to magnetic reconnection across the heliospheric current sheet [Moldwin et al., 1995, 2000] is supported by our observations of being small in scale size, lacking depressed proton temperature, and that they are found near the sector crossing. The vertical extent of the heliospheric current sheet is $\sim 10^{4} \mathrm{~km}$ [e.g., Winterhalter et al., 1994] and the average scale size of small-scale flux ropes is $\sim 10$ times this length scale. This is similar to the dimension of the magnetotail current sheet and the scale size of the tail flux ropes [e.g., Linton and Moldwin, 2009]. However, our results also indicate the source region is in the inner heliosphere. This does not rule out the possibility of a solar wind reconnection-type process creating these structures [Gosling, 2005], but it does suggest that most, if not all, are formed in the inner heliosphere.

[42] We believe our current results are most consistent with formation by (1) magnetic reconnection across the current sheet in the inner heliosphere and possibly (2) a formation mechanism; that small-scale flux ropes are remnants of the streamer belt blobs formed from disconnection [Wang et al., 1998]. The streamer belt blobs are observed in the slow wind and have a bipolar structure [Wang et al., 1998]. This would account for the lack of flux ropes during solar maximum when the helmet streamers are less confined to the solar equator. This hypothesis could also explain the expansion of the small-scale flux rope dimension from the inner to outer heliosphere. If they are created near the Sun and are overpressured flux ropes, then they could expand into the solar wind consistent with the observations. However, no one-to-one observation of a blob and a small-scale flux rope in the solar wind has yet been found.

\section{Appendix A}

[43] Table A1. 
Table A1. Small-Scale Solar Wind Flux Rope Database

\begin{tabular}{|c|c|c|}
\hline Spacecraft & Start Time (UT) & End Time (UT) \\
\hline Helios 1 & 29 Dec 19741333 & 29 Dec 19741450 \\
\hline Helios 1 & 4 Mar 19750219 & 4 Mar 19750247 \\
\hline Helios 1 & 19 Aug 19752019 & 19 Aug 19752055 \\
\hline Helios 2 & 4 Mar 19760520 & 4 Mar 19760631 \\
\hline Helios 2 & 11 Apr 19761919 & 11 Apr 19762122 \\
\hline Helios 2 & 22 Nov 19761812 & 22 Nov 19762000 \\
\hline Helios 1 & 5 Dec 19760725 & 5 Dec 19760833 \\
\hline Helios 1 & 3 Jan 19770906 & 3 Jan 19770956 \\
\hline Helios 1 & 11 Jan 19770509 & 11 Jan 19770539 \\
\hline Helios 1 & 15 Jan 19771517 & 15 Jan 19771555 \\
\hline Helios 1 & 27 Jan1977 0140 & 27 Jan 19770325 \\
\hline Helios 2 & 19 Feb 19772303 & 20 Feb 19770403 \\
\hline Helios 2 & 16 Mar 19771430 & 16 Mar 19771523 \\
\hline Helios 1 & 22 Mar 19771001 & 22 Mar 19771022 \\
\hline Helios 1 & 14 Apr 19771725 & 14 Apr 19772134 \\
\hline Helios 2 & 29 Oct 19771823 & 29 Oct1977 2002 \\
\hline Helios 1 & 6 Dec 19781302 & 06 Dec 19781349 \\
\hline Helios 1 & 19 Jan 19791832 & 19 Jan 19792019 \\
\hline Helios 2 & 25 Nov 19790912 & 25 Nov 19790957 \\
\hline Helios 2 & 30 Dec 19791732 & 30 Dec 19791956 \\
\hline Helios 1 & 2 Jan 19801313 & 2 Jan 19801632 \\
\hline Helios 1 & 16 Jan 19801220 & 16 Jan 19801422 \\
\hline Helios 1 & 14 May 19800815 & 14 May 19800958 \\
\hline IMP 8 & 29 May 19751510 & 29 May 19751552 \\
\hline IMP 8 & 7 Jun 19750752 & 7 Jun 19750919 \\
\hline IMP 8 & 18 Jan 19760548 & 18 Jan 19760654 \\
\hline IMP 8 & 22 Jan 19760419 & 22 Jan 19760514 \\
\hline IMP 8 & 26 Feb 19761712 & 26 Feb 19761750 \\
\hline IMP 8 & 21 Oct 19762057 & 21 Oct 19762145 \\
\hline IMP 8 & 7 Nov 19760004 & 07 Nov 19760421 \\
\hline IMP 8 & 14 Apr 19770723 & 14 Apr 19770904 \\
\hline IMP 8 & 27 Apr 19771051 & 27 Apr 19771217 \\
\hline IMP 8 & 25 Dec 19771852 & 25 Dec 19772104 \\
\hline IMP 8 & 2 Jan 19780430 & 2 Jan 19780508 \\
\hline IMP 8 & 19 Jan 19780107 & 19 Jan 19780449 \\
\hline IMP 8 & 16 Jun 19781541 & 16 Jun 19781719 \\
\hline IMP 8 & 20 Jul 19790426 & $20 \mathrm{Jul} 19790531$ \\
\hline IMP 8 & 20 Sep 19791443 & 20 Sep 19791737 \\
\hline IMP 8 & 15 Jan 19802230 & 16 Jan 19800037 \\
\hline IMP 8 & 24 Jan 19800840 & 24 Jan 19800933 \\
\hline IMP 8 & 21 Feb 19802102 & 22 Feb 19800128 \\
\hline IMP 8 & 19 Mar 19800617 & 19 Mar 19800745 \\
\hline IMP 8 & 28 Mar 19802232 & 29 Mar 19800012 \\
\hline IMP 8 & 6 May 19800937 & 6 May 19801109 \\
\hline IMP 8 & 31 Oct 19812018 & 31 Oct 19812232 \\
\hline IMP 8 & 19 May 19821825 & 19 May 19821918 \\
\hline IMP 8 & 6 Oct 19821236 & 06 Oct 19821642 \\
\hline IMP 8 & 31 Oct 19822234 & 1 Nov 19820113 \\
\hline IMP 8 & 24 Nov 19822241 & 25 Nov 19820053 \\
\hline IMP 8 & 3 Jan 19831832 & 3 Jan 19831859 \\
\hline IMP 8 & 11 May 19831739 & 11 May 19831831 \\
\hline IMP 8 & 24 Jul 19830945 & 24 Jul 19831214 \\
\hline IMP 8 & 6 Oct 19830931 & 6 Oct 19831206 \\
\hline IMP 8 & 14 Jan 19842239 & 14 Jan 19842247 \\
\hline IMP 8 & 15 Jan 19852140 & 15 Jan 19852322 \\
\hline IMP 8 & 21 Mar 19851629 & 21 Mar 19851938 \\
\hline IMP 8 & 22 Mar 19852154 & 23 Mar 19850014 \\
\hline IMP 8 & 30 Mar 19851346 & 30 Mar 19851437 \\
\hline IMP 8 & 31 Mar 19851019 & 31 Mar 19851223 \\
\hline IMP 8 & 25 Apr 19850658 & 25 Apr 19850840 \\
\hline IMP 8 & 6 May 19850343 & 6 May 19850419 \\
\hline IMP 8 & 11 May 19850746 & 11 May 19850851 \\
\hline IMP 8 & 1 Jun 19850640 & 1 Jun 19850802 \\
\hline IMP 8 & 2 Jun 19852051 & 2 Jun 19852144 \\
\hline IMP 8 & 4 Jun 19852047 & 5 Jun 19850053 \\
\hline IMP 8 & 1 Nov 19851807 & 1 Nov 19852001 \\
\hline IMP $8^{\mathrm{a}}$ & 13 Apr 19860724 & 13 Apr 19861524 \\
\hline IMP 8 & 22 Apr 19860904 & 22 Apr 19861043 \\
\hline IMP 8 & 24 Jul 19862107 & 24 Jul 19862310 \\
\hline IMP 8 & 5 Oct 19860827 & 5 Oct 19861035 \\
\hline IMP 8 & 18 Oct 19860741 & 18 Oct 19860824 \\
\hline IMP 8 & 10 Dec 19861855 & 10 Dec 19861951 \\
\hline
\end{tabular}

Table A1. (continued)

\begin{tabular}{|c|c|c|}
\hline Spacecraft & Start Time (UT) & End Time (UT) \\
\hline IMP 8 & 12 Jan 19870929 & 12 Jan 19871102 \\
\hline IMP 8 & 6 Feb 19871020 & 6 Feb 19871113 \\
\hline IMP 8 & 30 Mar 19872014 & 31 Mar 19870219 \\
\hline IMP 8 & 2 Nov 19871800 & 2 Nov 19871900 \\
\hline IMP 8 & 11 Feb 19882018 & 11 Feb 19882145 \\
\hline IMP 8 & 6 Mar 19881755 & 6 Mar 19882026 \\
\hline IMP 8 & 26 Apr 19882231 & 27 Apr 19880116 \\
\hline IMP 8 & 3 Mar 19890529 & 03 Mar 19890711 \\
\hline IMP 8 & $10 \mathrm{Jul} 19890346$ & 10 Jul 19890640 \\
\hline IMP 8 & 6 Feb 19901820 & 06 Feb 19902213 \\
\hline IMP 8 & 25 Jan 19911616 & 25 Jan 19911714 \\
\hline IMP 8 & 24 Apr 19922331 & 25 Apr 19920022 \\
\hline IMP 8 & 15 Aug 19920206 & 15 Aug 19920649 \\
\hline IMP 8 & 9 Sep 19921050 & 09 Sep 19921434 \\
\hline IMP 8 & 21 Dec 19922043 & 21 Dec 19922206 \\
\hline IMP 8 & 11 Feb 19930030 & 11 Feb 19930137 \\
\hline IMP 8 & 15 Aug 19942116 & 15 Aug 19942343 \\
\hline Wind & 14 Jan 19950547 & 14 Jan 19950853 \\
\hline Wind & 21 Feb 19951921 & 21 Feb 19952116 \\
\hline Wind & 24 Mar 19950250 & 24 Mar 19950648 \\
\hline Wind & 24 Mar 19951123 & 24 Mar 19951623 \\
\hline Wind & 2 May 19950447 & 2 May 19950540 \\
\hline Wind & 16 May 19950111 & 16 May 19950917 \\
\hline Wind & 7 Jun 19950938 & 7 Jun 19951836 \\
\hline Wind & 19 Jun 19950110 & 19 Jun 19950206 \\
\hline Wind & 4 Jul 19950259 & 4 Jul 19950500 \\
\hline Wind & 11 Aug 19950418 & 11 Aug 19950557 \\
\hline Wind & 15 Aug 19951418 & 15 Aug 19951719 \\
\hline Wind & 17 Aug 19952027 & 18 Aug 19950157 \\
\hline Wind & 19 Sep 19951849 & 19 Sep 19951946 \\
\hline Wind & 20 Sep 19951259 & 20 Sep 19951414 \\
\hline Wind & 21 Sep 19950252 & 21 Sep 19950454 \\
\hline Wind & 27 Sep 19951349 & 27 Sep 19952130 \\
\hline Wind & 24 Oct 19951309 & 24 Oct 19951409 \\
\hline Wind & 27 Oct 19951035 & 27 Oct 19951138 \\
\hline Wind & 3 Dec 19950402 & 3 Dec 19950541 \\
\hline Wind & 13 Mar 19960924 & 13 Mar 19961044 \\
\hline Wind & 16 Mar 19962227 & 17 Mar 19960228 \\
\hline Wind & 19 Mar 19960902 & 19 Mar 19960956 \\
\hline Wind & 11 Apr 19961314 & 11 Apr 19961447 \\
\hline Wind & 14 Apr 19961052 & 14 Apr 19961228 \\
\hline Wind & 14 May 19962154 & 14 May 19962313 \\
\hline Wind & 11 Aug 19962059 & 12 Aug 19960136 \\
\hline Wind & 23 Aug 19960243 & 23 Aug 19960550 \\
\hline Wind & 29 Aug 19960121 & 29 Aug 19960211 \\
\hline Wind & 11 Nov 19961636 & 11 Nov 19961952 \\
\hline Wind & 28 Jan 19970217 & 28 Jan 19970547 \\
\hline Wind & 23 Mar 19971212 & 23 Mar 19971339 \\
\hline Wind & 9 May 19970323 & 9 May 19970846 \\
\hline Wind & 24 Jul 19970727 & 24 Jul 19970907 \\
\hline Wind & 2 Sep 19970231 & 2 Sep 19970654 \\
\hline Wind & 6 Jan 19981959 & 6 Jan 19982215 \\
\hline Wind & 16 Jan 19981128 & 16 Jan 19981311 \\
\hline Wind/ACE ${ }^{b}$ & 1 Feb 19980050 & 1 Feb 19980626 \\
\hline Wind/ACE & 23 Feb 19981534 & 23 Feb 19981748 \\
\hline Wind/ACE & 27 Feb 19980534 & 27 Feb 19980846 \\
\hline Wind/ACE & 20 Mar 19981442 & 20 Mar 19981712 \\
\hline Wind/ACE & 10 Jun 19982236 & 10 Jun 19982347 \\
\hline Wind/ACE & 10 Jul 19982246 & 11 Jul 19980858 \\
\hline Wind/ACE & 6 Nov 19982158 & 6 Nov 19982324 \\
\hline Wind/ACE & 3 Feb 19990924 & 3 Feb 19991201 \\
\hline ACE & 3 Feb 19991206 & 3 Feb 19991351 \\
\hline ACE & 4 Feb 19991010 & 4 Feb 19991318 \\
\hline Wind/ACE & 11 Feb 19990011 & 11 Feb 19990333 \\
\hline $\mathrm{ACE}$ & 25 Mar 19991608 & 25 Mar 19992259 \\
\hline $\mathrm{ACE}$ & 23 Apr 19990537 & 23 Apr 19990829 \\
\hline Wind/ACE & 24 May 19990900 & 24 May 19991045 \\
\hline Wind/ACE & 27 Jun 19991117 & 27 Jun 19991156 \\
\hline Wind/ACE & 6 Aug 19991029 & 6 Aug 19991225 \\
\hline Wind/ACE & 11 Aug 19990010 & 11 Aug 19990416 \\
\hline $\mathrm{ACE}$ & 17 Nov 19991608 & 17 Nov 19991659 \\
\hline Wind/ACE & 28 Dec 19990626 & 28 Dec 19990807 \\
\hline
\end{tabular}


Table A1. (continued)

\begin{tabular}{|c|c|c|}
\hline Spacecraft & Start Time (UT) & End Time (UT) \\
\hline Wind/ACE & 27 Apr 20001850 & 27 Apr 20002347 \\
\hline Wind/ACE & 19 Jul 20002117 & 20 Jul 20000043 \\
\hline Wind/ACE & 12 Aug 20000220 & 12 Aug 20000526 \\
\hline Wind/ACE & 1 Oct 20001924 & 1 Oct 20002242 \\
\hline Wind/ACE & 29 Dec 20000115 & 29 Dec 20000326 \\
\hline ACE & 18 Feb 20012136 & 18 Feb 20012328 \\
\hline Wind/ACE & 31 Mar 20010606 & 31 Mar 20011231 \\
\hline Wind & 18 Мay 20010952 & 18 May 20011323 \\
\hline Wind/ACE & 9 Jun 20010314 & 9 Jun 20010500 \\
\hline Wind/ACE & 10 May 20021709 & 10 May 20022006 \\
\hline Wind/ACE & 7 Jun 20021918 & 7 Jun 20022318 \\
\hline Wind/ACE & 19 Jun 20020249 & 19 Jun 20020340 \\
\hline Wind/ACE & 7 Jul 20030322 & 7 Jul 20030442 \\
\hline Wind/ACE & 9 Jun 20040649 & 9 Jun 20040915 \\
\hline Wind/ACE & 9 Jul 20040535 & 9 Jul 20040922 \\
\hline Wind/ACE & 9 Aug 20040201 & 9 Aug 20040648 \\
\hline Wind/ACE & 14 Sep 20041634 & 14 Sep 20042127 \\
\hline Wind/ACE & 8 Oct 20040705 & 8 Oct 20040840 \\
\hline Wind/ACE & 7 Jan 20051155 & 7 Jan 20051357 \\
\hline Wind/ACE & 7 Jan 20052046 & 8 Jan 20050707 \\
\hline Wind & 10 Jan 20052255 & 10 Jan 20052341 \\
\hline Wind & 14 Jan 20051722 & 14 Jan 20051959 \\
\hline Wind/ACE & 5 Mar 20050727 & 5 Mar 20050929 \\
\hline Wind/ACE & 31 Маy 20051925 & 31 May 20052218 \\
\hline Wind/ACE & 24 Aug 20050823 & 24 Aug 20051027 \\
\hline Wind/ACE & 31 Aug 20051534 & 31 Aug 20051701 \\
\hline Wind/ACE & 30 Sep 20051041 & 30 Sep 20051231 \\
\hline Wind/ACE & 14 Jan 20061739 & 14 Jan 20061931 \\
\hline Wind/ACE & 22 Jan 20061249 & 22 Jan 20061502 \\
\hline Wind/ACE & 19 Feb 20060351 & 19 Feb 20060719 \\
\hline Wind/ACE & 8 Apr 20060733 & 8 Apr 20060919 \\
\hline Wind/ACE & 30 May 20061339 & 30 May 20061605 \\
\hline Wind/ACE & 24 Jun 20062309 & 25 Jun 20060142 \\
\hline Wind & 26 Jul 20061727 & $26 \mathrm{Jul} 20061854$ \\
\hline Wind/ACE & 12 Sep 20060128 & 12 Sep 20060515 \\
\hline Wind/ACE & 28 Jan 20070017 & 28 Jan 20070316 \\
\hline Wind/ACE & 19 Feb 20071029 & 19 Feb 20071212 \\
\hline Wind/ACE & 1 Mar 20071611 & 1 Mar 20071654 \\
\hline ACE & 12 Mar 20071229 & 12 Mar 20071314 \\
\hline Wind/ACE & 18 Apr 20072118 & 18 Apr 20072234 \\
\hline Wind/ACE & 15 Jul 20070117 & 15 Jul 20070157 \\
\hline Wind/ACE & 30 Sep 20072054 & 30 Sep 20072205 \\
\hline Wind/ACE & 30 Nov 20071159 & 30 Nov 20071414 \\
\hline Ulysses & 3 Nov 19900731 & 3 Nov 19900812 \\
\hline Ulysses & 28 Jan 19911003 & 28 Jan 19911623 \\
\hline Ulysses & 24 Apr 19910550 & 24 Apr 19911015 \\
\hline Ulysses & 25 Apr 19911416 & 25 Apr 19911642 \\
\hline Ulysses & 8 May 19911335 & 8 May 19911621 \\
\hline Ulysses & 27 Jun 19912237 & 28 Jun 19910113 \\
\hline Ulysses & 5 Nov 19910540 & 5 Nov 19911137 \\
\hline Ulysses & 27 Apr 19920032 & 27 Apr 19920339 \\
\hline Ulysses & 23 May 19920707 & 23 May 19921158 \\
\hline Ulysses & 21 Jun 19922242 & 22 Jun 19920447 \\
\hline Ulysses & 15 Jul 19920821 & 15 Jul 19921015 \\
\hline Ulysses & 1 Aug 19922038 & 2 Aug 19920009 \\
\hline Ulysses & 25 Jan 19981613 & 26 Jan 19980034 \\
\hline Ulysses & 20 Jan 20040940 & $20 \mathrm{Jan} 20041035$ \\
\hline Ulysses & 16 Jun 20041329 & 16 Jun 20041622 \\
\hline Ulysses & 13 Jul 20040735 & $13 \mathrm{Jul} 20041630$ \\
\hline Ulysses & 4 Aug 20040306 & 4 Aug 20040830 \\
\hline Ulysses & 28 Nov 20040605 & 28 Nov 20040755 \\
\hline Ulysses & 10 Dec 20040745 & 10 Dec 20041139 \\
\hline Ulysses & 27 Jan 20051842 & 27 Jan 20052002 \\
\hline
\end{tabular}

${ }^{a}$ This flux rope had a small data gap ( $5 \%$ of total duration) that was linearly interpolated across.

${ }^{b}$ When the flux rope was found by Wind and ACE, the time at Wind was used.
[44] Acknowledgments. We would like to thank the magnetic field and plasma data set teams for their careful work: PIs Ness, Neubauer, and Rosenbauer for Helios 1 and Helios 2 data sets; PIs Lepping and Lazarus for the IMP 8 data set; the Wind spacecraft MFI (PI: Lepping) and SWE (PIs: Ogilvie and Lazarus) teams; the ACE MAG (PI: Ness) and SWEPAM (Los Alamos) teams; and the Ulysses magnetic field team (PI: Balogh) and the plasma team SWOOPS (PI: Bame). We also thank the referees for their helpful comments that greatly improved the paper. We would like to thank the National Space Science Data Center and the Space Physics Data Facility for making all the data sets publicly available. We thank J. Weygand for the use of the solar wind data set he has carefully compiled. This work was supported by NASA SR\&T (NAG5-12823) and a NASA Goddard Space Flight Center GSRP Fellowship

[45] Zuyin Pu thanks Ian Richardson and another reviewer for their assistance in evaluating this paper.

\section{References}

Balogh, A., T. J. Beek, R. J. Forsyth, P. C. Hedgecock, R. J. Marquedant, E. J. Smith, D. J. Southwood, and B. T. Tsurutani (1992), The magnetic field investigation on the ULYSSES mission-Instrumentation and preliminary scientific results, Astron. Astrophys. Suppl. Ser., 92, 221-236.

Bame, S. J., D. J. McComas, B. L. Barraclough, J. L. Phillips, K. J. Sofaly, J. C. Chavez, B. E. Goldstein, and R. K. Sakurai (1992), The ULYSSES solar wind plasma experiment, Astron. Astrophys. Suppl. Ser., 92, 237265

Bothmer, V., and D. M. Rust (1997), The field configuration of magnetic clouds and the solar cycle, in Coronal Mass Ejections, Geophys. Monogr. Ser., vol. 99, edited by N. Crooker et al., pp. 139-146, AGU, Washington, D. C.

Bothmer, V., and R. Schwenn (1998), The structure and origin of magnetic clouds in the solar wind, Ann. Geophys., 16, 1-24.

Brueckner, G. E., R. A. Howard, M. J. Kooman, C. M. Korendyke, D. J. Michels, J. D. Moses, D. G. Socker, K. P. Dere, P. L. Lamy, A. Llebaria, et al. (1995), The large angle spectroscopic coronagraph (LASCO), Sol. Phys., 162, 357-402.

Bruno, R., B. Bavassano, and U. Villante (1985), Evidence for long period Alfvén waves in the inner solar system, J. Geophys. Res., 90(A5), 43734377, doi:10.1029/JA090iA05p04373.

Burlaga, L., E. Sittler, F. Mariani, and R. Schwenn (1981), Magnetic loop behind an interplanetary shock-Voyager, Helios, and IMP 8 observations, J. Geophys. Res., 86, 6673-6684.

Burlaga, L. F. (1988), Magnetic clouds and force-free fields with constant alpha, J. Geophys. Res., 93(A7), 7217-7224.

Burlaga, L. F., and K. W. Behannon (1982), Magnetic clouds: Voyage observations between 2 and 4 AU, Sol. Phys., 81, 181-192, doi:10.1007/ BF00151989.

Burlaga, L. F., and J. M. Turner (1976), Microscale "Alfvén waves" in the solar wind at $1 \mathrm{AU}, J$. Geophys. Res., 81(1) 73-77, doi:10.1029/ JA081i001p00073.

Cane, H. V., and I. G. Richardson (2003), Interplanetary coronal mass ejections in the near-Earth solar wind during 1996-2002, J. Geophys. Res., 108(A4), 1156, doi:10.1029/2002JA009817.

Cartwright, M. L., and M. B. Moldwin (2008), Comparison of small-scale flux rope magnetic properties to large-scale magnetic clouds: Evidence for reconnection across the HCS?, J. Geophys. Res., 113, A09105, doi:10.1029/2008JA013389.

Farrugia, C. F., V. A. Osherovich, and L. F. Burlaga (1995), The magnetic flux rope versus the spheromak as models for interplanetary magnetic clouds, J. Geophys. Res., 100(A7), 12,293-12,306, doi:10.1029/ 95JA00272.

Feng, H. Q., D. J. Wu, and J. K. Chao (2007), Size and energy distributions of interplanetary magnetic flux ropes, J. Geophys. Res., 112, A02102, doi:10.1029/2006JA011962.

Feng, H. Q., D. J. Wu, C. C. Lin, J. K. Chao, L. C. Lee, and L. H. Lyu (2008), Interplanetary small-and intermediate-sized magnetic flux ropes during 1995-2005, J. Geophys. Res., 113, A12105, doi:10.1029/ 2008JA013103

Forsyth, R. J., V. Bothmer, C. Cid, N. U. Crooker, T. S. Horbury, K. Kecskemety, B. Klecker, J. A. Linker, D. Odstrcil, M. J. Reiner, et al. (2006), ICMEs in the inner heliosphere: Origin, evolution and propagation effects. Report of Working Group G, Space Sci. Rev., 123(1-3), 383, doi:10.1007/s11214-006-9022-0.

Goldstein, H. (1983), On the field configuration in magnetic clouds, in Solar Wind Five, edited by M. Neugebauer, NASA Conf. Publ., CP2280, 731-733.

Gosling, J. T. (2005), Magnetic reconnection in the solar wind: A brief overview, in Proceedings of the Solar Wind 11/SOHO 16 "Connecting 
Sun and Heliosphere" Conference (ESA SP-592), edited by B. Fleck et al., p. 249, 12-17 June 2005, Whistler, Canada.

Henke, T., J. Woch, R. Schwenn, U. Mall, G. Gloeckler, R. von Steiger, R. J. Forsyth, and A. Balogh (2001), Ionization state and magnetic topology of coronal mass ejections, J. Geophys. Res., 106(A6), doi:10.1029/ 2000JA900176.

Jian, L., C. T. Russell, J. G. Luhmann, R. M. Skoug, and J. T. Steinberg (2008), Stream interactions and interplanetary coronal mass ejections at 5.3 AU near the solar ecliptic plane, Solar Phys., 250(2), 375-402, doi:10.1007/s11207-008-904-x.

Klein, L. W., and L. F. Burlaga (1982), Interplanetary magnetic clouds at 1 AU, J. Geophys. Res., 87(A2), 613-624, doi:10.1029/ JA087iA02p00613.

Lepping, R. P., and C.-C. Wu (2007), On the variation of interplanetary magnetic cloud type through solar cycle 23: Wind events, J. Geophys. Res., 112, A10103, doi:10.1029/2006JA012140.

Lepping, R. P., L. F. Burlaga, and J. A. Jones (1990), Magnetic field structure of interplanetary magnetic clouds at $1 \mathrm{AU}, J$. Geophys. Res., 95(A8), 11,957-11,965, doi:10.1029/JA095iA08p11957.

Lepping, R. P., et al. (1995), The WIND magnetic field investigation, Space Sci. Rev., 71(1-4), 207-209, doi:10.1007/BF00751330.

Lepping, R. P., A. Szabo, M. Peredo, and J. T. Hoeksema (1996), Largescale properties and solar connection of the heliospheric current and plasma sheets: WIND observations, Geophys. Res. Lett., 23(10), 11991202, doi:10.1029/96GL00658.

Lepping, R. P., D. B. Berdichevsky, C.-C. Wu, A. Szabo, T. Narock, F. Mariani, A. J. Lazarus, and A. J. Quivers (2006), A summary of WIND magnetic clouds for years 1995-2003: Model-fitted parameters, associated errors and classifications, Ann. Geophys., 24(1), 215-245, doi:10.5194/angeo-24-215-2006.

Linton, M. G., and M. B. Moldwin (2009), A comparison of the formation and evolution of magnetic flux ropes in solar coronal mass ejections and magnetotail plasmoids, J. Geophys. Res., 114, A00B09, doi:10.1029/ 2008JA013660

Lundquist, S. (1950), Magnetohydrostatic fields, Ark. Phys, 2, 361-365.

Lynch, B. J., J. R. Gruesbeck, and T. H. Zurbuchen (2005), Solar cycledependent helicity transport by magnetic clouds, J. Geophys. Res., 110 A08107, doi:10.1029/2005JA011137.

Mandrini, C. H., et al. (2005), The smallest source region of an interplanetary magnetic cloud: A mini-sigmoid, Adv. Space Sci., 36, 1579-1586.

McComas, D. J., S. J. Bame, P. Barker, W. C. Feldman, J. L. Phillips, P. Riley, and J. W. Griffee (1998), Solar wind electron proton alpha monitor (SWEPAM) for the Advanced Composition Explorer, Space Sci. Rev., 86, 563, doi:10.1023/A:1005040232597.

Moldwin, M. B., and W. J. Hughes (1992), On the formation and evolution of plasmoids - A survey of ISEE 3 Geotail data, J. Geophys. Res., 97(A12), 19,259-19,282.

Moldwin, M. B., J. L. Phillips, J. T. Gosling, E. E. Scime, D. J. McComas, S. J. Bame, A. Balogh, and R. J. Forsyth (1995), Ulysses observations of a noncoronal mass ejection flux rope: Evidence of interplanetary magnetic reconnection, J. Geophys. Res., 100(10), 19,903-19,910 doi:10.1029/95JA01123.

Moldwin, M. B., S. Ford, R. Lepping, J. Slavin, and A. Szabo (2000), Small-scale magnetic flux ropes in the solar wind, Geophys. Res. Lett. 27(1), 57-60, doi:10.1029/1999GL010724.

Mulligan, T., C. T. Russell, and J. Luhmann (1998), Solar cycle evolution of the structure of magnetic clouds in the inner heliosphere, Geophys. Res. Lett., 25(15), 2959-2962, doi:10.1029/98GL01302.
Mulligan, T. L. (2002), The structure of interplanetary coronal mass ejections and their solar origins, Ph.D. dissertation, 370 pp., Univ. of Calif., Los Angeles.

Ogilvie, K. W., D. J. Chornay, R. J. Fritzenreiter, F. Hunsaker, J. Keller, J. Lobell, G. Miller, J. D. Scudder, E. C. Sittler Jr., R. B. Torbert, et al. (1995), SWE, a comprehensive plasma instrument for the WIND, Space Sci. Rev, 71, 55, doi: 10.1007/BF00751326.

Osherovich, V., J. Fainberg, and R. Stone (1999), Multi-tube model for interplanetary magnetic clouds, Geophys. Res. Lett., 26(3), 401-404, doi:10.1029/1998GL900306.

Richardson, I. G., and H. V. Cane (2004), The fraction of interplanetary coronal mass ejections that are magnetic clouds: Evidence for a solar cycle variation, Geophys. Res. Lett., 31, L18804, doi:10.1029 2004GL020958.

Rosenbauer, H., R. Schwenn, E. Marsh, B. Meyer, H. Miggenrieder, M. D. Montgomery, K. H. Mulhauser, W. Phillip, W. Voges, and S. M. Zink (1977), A survey on initial results of the Helios plasma experiment, J. Geophys. Res., 42, 561-580.

Scearce, C., S. Cantarano, N. Ness, F. Mariani, R. Terenzi, and L. Burlaga (1975), Rome-GSFC magnetic field experiment for Helios A and B (E3), Raumfahrtforschung, 19, 237-240.

Smith, C. W., J. L'Heureux, N. F. Ness, M. H. Acuña, L. F. Burlaga, and J. Scheifele (1998), The ACE magnetic fields experiment, Space Sci. Rev., 86, 613, doi:10.1023/A:1005092216668

Smith, E. J., A. Balogh, M. Neugebauer, and D. McComas (1995), Ulysses observations of Alfvén waves in the southern and northern solar hemispheres, Geophys. Res. Lett., 22(23), 3381-3384, doi:10.1029 95GL03268.

Sonnerup, B. U. O., and L. J. Cahill (1967), Magnetopause structure and attitude from Explorer 12 observations, J. Geophys. Res., 72(1), 171-183, doi:10.1029/JZ072i001p00171.

Vandas, M., S. Fisher, P. Pelant, and A. Geranios (1993), Spheriodal models of magnetic clouds and their comparison with spacecraft measurements, J. Geophys. Res., 98(A7), 11,467-11,475, doi:10.1029/93JA00055.

Wang, Y.-M., N. R. Sheeley Jr., J. H. Walters, G. E. Brueckner, R. A Howard, and D. J. Michels (1998), Origin of streamer material in the outer corona, APJ, 498, L165-L168.

Winterhalter, D., E. J. Smith, M. E. Burton, N. Murphy, and D. J. McComas (1994), The heliospheric plasma sheet, J. Geophys. Res., 99(A4), 66676680, doi:10.1029/93JA03481.

Wu, C.-C., and R. P. Lepping (2007), Comparison of the Characteristics of Magnetic Clouds and Magnetic Cloud-like Structures for the Events of 1995-2003, Solar Phys., 242, 159-165, doi:10.1007/s11207-007-0323-6.

Yashiro, S., N. Gopalswamy, G. Michalek, O. C. St. Cyr, S. P. Plunkett, N. B. Rich, and R. A. Howard (2004), A catalog of white light coronal mass ejections observed by the SOHO spacecraft, J. Geophys. Res., 109, A07105, doi: $10.1029 / 2003$ JA010282.

M. L. Cartwright, Space Sciences Laboratory, University of California, 7 Gauss Way, Berkeley, CA 94720-7450, USA. (mcartwright@ssl. berkeley.edu)

M. B. Moldwin, Department of Atmospheric, Oceanic, and Space Sciences, University of Michigan, Space Research Bldg., 2455 Hayward St., Ann Arbor, MI 48109-2143, USA. 